H. Yamashita

Nagoya Math. J.

Vol. 156 (1999), 85-108

\title{
ON THE RANK OF THE FIRST RADICAL LAYER OF A P-CLASS GROUP OF AN ALGEBRAIC NUMBER FIELD
}

\author{
HIROSHI YAMASHITA
}

\begin{abstract}
Let $p$ be a prime number. Let $M$ be a finite Galois extension of a finite algebraic number field $k$. Suppose that $M$ contains a primitive $p$ th root of unity and that the $p$-Sylow subgroup of the Galois group $G=\operatorname{Gal}(M / k)$ is normal. Let $K$ be the intermediate field corresponding to the $p$-Sylow subgroup. Let $\mathfrak{g}=\operatorname{Gal}(K / k)$. The $p$-class group $\mathcal{C}$ of $M$ is a module over the group ring $\mathbf{Z}_{p} G$, where $\mathbf{Z}_{p}$ is the ring of $p$-adic integers. Let $J$ be the Jacobson radical of $\mathbf{Z}_{p} G \cdot \mathcal{C} / J \mathcal{C}$ is a module over a semisimple artinian ring $\mathbf{F}_{p} \mathfrak{g}$. We study multiplicity of an irreducible representation $\Phi$ apperaring in $\mathcal{C} / J \mathcal{C}$ and prove a formula giving this multiplicity partially. As application to this formula, we study a cyclotomic field $M$ such that the minus part of $\mathcal{C}$ is cyclic as a $\mathbf{Z}_{p} G$ module and a CM-field $M$ such that the plus part of $\mathcal{C}$ vanishes for odd $p$.

To show the formula, we apply theory of central extensions of algebraic number field and study global and local Kummer duality between the genus group and the Kummer radical for the genus field with respect to $M / K$.
\end{abstract}

\section{Introduction}

Let $k$ be a finite extension of $\mathbf{Q}$ and $M$ be a finite Galois extension of $k$ with a Galois group $G$. Let $p$ be a prime number. Let $H$ be the $p$-Hilbert class field of $M$. The Galois group $\operatorname{Gal}(H / M)$ is isomorphic to the $p$-Sylow subgroup $\mathcal{C}$ of the ideal class group of $M$. Let $\mathbf{F}_{p}$ be the field of $p$-elements and denote by $\mathbf{F}_{p} G$ the group ring of $G$ over $\mathbf{F}_{p}$. Let $J$ be the Jacobson radical of $\mathbf{F}_{p} G . \mathcal{C} \otimes \mathbf{F}_{p}$ is an $\mathbf{F}_{p} G$-module and $\mathcal{C} \otimes \mathbf{F}_{p} / J \mathcal{C} \otimes \mathbf{F}_{p}$ is called the first radical layer of $\mathcal{C} \otimes \mathbf{F}_{p}$.

We suppose that the $p$-Sylow group $G_{p}$ of $G$ is a normal subgroup. Let $K$ be the intermediate field of $M / k$ corresponding to $G_{p} . K$ is a Galois extension of $k$ such that $p \nmid[K: k]$. Denote by $\mathfrak{g}$ the Galois group of $K / k$. The abelian $p$-genus field of $M / K$ is the compositum $H^{\mathrm{ab}} M$, where $H^{\mathrm{ab}}$ is the maximal abelian subfield of $H / K$. The central $p$-genus field $H^{\text {cent }}$ is the intermediate field of $H / M$ corresponding to $\prod_{\sigma \in G_{p}} \mathcal{C}^{\sigma-1}$. We shall

Received August 15, 1996. 
show that $J$ is generated by $\left\{g-1: g \in G_{p}\right\}$. Therefore, $\mathcal{C} \otimes \mathbf{F}_{p} / J \mathcal{C} \otimes \mathbf{F}_{p} \cong$ $\operatorname{Gal}\left(H^{\text {cent }} / M\right) \otimes \mathbf{F}_{p}$ and $\mathbf{F}_{p} G / J \cong \mathbf{F}_{p} \mathfrak{g}$. This means that $\operatorname{Gal}\left(H^{\text {cent }} / M\right) \otimes \mathbf{F}_{p}$ is an $\mathbf{F}_{p} \mathfrak{g}$-module which is isomorphic to the first radical layer.

$\mathbf{F}_{p} \mathfrak{g}$ is a semisimple artinian ring, because of $p \nmid(\mathfrak{g}: 1)$, (c.f. Theorem 3.14, Curtis-Reiner [2]). By Theorem 1.3.5, Benson [1], we have an isomorphism

$$
\mathbf{F}_{p} \mathfrak{g} \cong \bigoplus_{i=1}^{r} M_{n_{i}}\left(\Delta_{i}\right)
$$

of rings, where $\Delta_{i}$ is a division ring. Let $L_{i}$ be the minimal left ideal of $M_{n_{i}}\left(\Delta_{i}\right) . \mathbf{F}_{p} \mathfrak{g}$ has exactly $r$ isomorphism classes of irreducible modules $L_{i}$, $i=1, \cdots, r$. Denote by $\Phi_{i}$ the irreducible character afforded with $L_{i}$. Put $\mathfrak{B}(\mathfrak{g})=\left\{\Phi_{i}: 1 \leq i \leq r\right\}$. This set is called a basic set of irreducible $\mathbf{F}_{p^{-}}$ characters of $\mathfrak{g}$. These irreducible characters are linearly independent over $\mathbf{F}_{p}$, (c.f., Lemma 3.3, Chapter 19, Karpilovsky [5]). Let $1_{i}$ be the inverse image of the identity matrix of $M_{n_{i}}\left(\Delta_{i}\right)$ with the above isomorphism. We see

$$
1=\sum_{i=1}^{r} 1_{i}, \quad 1_{i} 1_{j}=\delta_{i, j} 1_{i} .
$$

When an irreducible character $\Phi$ is given, there is $\Phi_{i}$ such that $\Phi_{i}=\Phi$. Hence, we write $\Delta_{\Phi}, L_{\Phi}, 1_{\Phi}$ for $\Delta_{i}, L_{i}, 1_{i}$. We also write $d_{\Phi}$ for $n_{i}$.

There is an integer $a_{\Phi} \geq 1$ such that

$$
1_{\Phi} \operatorname{Gal}\left(H^{\text {cent }} / M\right) \otimes \mathbf{F}_{p} \cong L_{\Phi}^{a_{\Phi}} .
$$

We shall give a formula describing the value of $a_{\Phi}$ in the present paper. This will be done in Theorem 9 in $\S 4$. We are able to determine for what $M$ and $\Phi$ the value of $a_{\Phi}$ equals 0 or 1 . The number of generators of $\mathcal{C}$ over $\mathbf{Z}_{p} G$ is obtained from the values of $a_{\Phi}$, where $\mathbf{Z}_{p} G$ denotes the group ring of $G$ over the ring $\mathbf{Z}_{p}$ of $p$-adic integers. Suppose $p$ is odd. When $k=\mathbf{Q}$ and $M$ is a cyclotomic field and if $a_{\Phi} \leq 1$ for every $\Phi$ such that $\tau 1_{\Phi} \neq 1_{\Phi}$, we have $\mathcal{C}^{\tau-1} \cong(\tau-1) \mathbf{Z}_{p} G /(\tau-1) S$, where $\tau$ is the complex conjugation and $S$ is an ideal generated by the Stickelberger elements (c.f. Sinnott [9]). In general, it is equivalent to $\mathcal{C}=0$ that $a_{\Phi}$ vanishes for every $\Phi$. We are able to apply this to study the Greenberg conjecture (c.f. Greenberg [3]). We could obtain a criterion whether the conjecture holds in its "trivial case".

We denote by $\operatorname{dim} X$ the dimension over $\mathbf{F}_{p}$ for an $\mathbf{F}_{p^{-}}$module $X$. The outline of our argument is as follows. Put $M^{\mathrm{ab}}=M \cap H^{\mathrm{ab}}$. There is a 
non-negative integer $\theta(\Phi)$ such that

$$
a_{\Phi}=\frac{1}{\operatorname{dim} L_{\Phi}} \operatorname{dim} 1_{\Phi} \operatorname{Gal}\left(H^{\mathrm{ab}} / M^{\mathrm{ab}}\right) \otimes \mathbf{F}_{p}+\theta(\Phi) .
$$

We have the following inequality:

$$
\begin{aligned}
& \operatorname{dim} 1_{\Phi} \operatorname{Gal}\left(H^{\mathrm{ab}} / K\right) \otimes \mathbf{F}_{p} \geq \operatorname{dim} 1_{\Phi} \operatorname{Gal}\left(H^{\mathrm{ab}} / M^{\mathrm{ab}}\right) \otimes \mathbf{F}_{p} \\
\geq & \operatorname{dim} 1_{\Phi} \operatorname{Gal}\left(H^{\mathrm{ab}} / K\right) \otimes \mathbf{F}_{p}-\operatorname{dim} 1_{\Phi} \operatorname{Gal}\left(M^{\mathrm{ab}} / K\right) \otimes \mathbf{F}_{p} .
\end{aligned}
$$

Hence, if $1_{\Phi} \operatorname{Gal}\left(M^{\mathrm{ab}} / K\right) \otimes \mathbf{F}_{p}=0$, we have

$$
\operatorname{dim} 1_{\Phi} \operatorname{Gal}\left(H^{\mathrm{ab}} / K\right) \otimes \mathbf{F}_{p}=\operatorname{dim} 1_{\Phi} \operatorname{Gal}\left(H^{\mathrm{ab}} / M^{\mathrm{ab}}\right) \otimes \mathbf{F}_{p} .
$$

We shall obtain a formula of the value of

$$
\operatorname{dim} 1_{\Phi} \operatorname{Gal}\left(H^{\mathrm{ab}} / K\right) \otimes \mathbf{F}_{p}
$$

by studying the representation of $\mathfrak{g}$ on $\operatorname{Gal}\left(H^{\mathrm{ab}} / K\right) \otimes \mathbf{F}_{p}$. In particular, when $G=G_{p} \times \mathfrak{g}$, we have $\theta(\Phi)=0$ and $\operatorname{dim} 1_{\Phi} \operatorname{Gal}\left(M^{\mathrm{ab}} / K\right) \otimes \mathbf{F}_{p}=0$ if $\Phi$ is not the unit character $\varepsilon$.

\section{$\S 1$. The $p$-genus and central $p$-genus fields}

The Jacobson radical $J$ of $\mathbf{F}_{p} G$ is the intersection of every maximal left ideal, which is a two-sided ideal of $\mathbf{F}_{p} G$. Put $R=\mathbf{F}_{p} G / J$.

LEMMA 1. J is generated by $\left\{g-1: g \in G_{p}\right\}$, and hence, $R \cong \mathbf{F}_{p} \mathfrak{g}$.

Proof. Let $\mathbf{F}_{p} G \rightarrow \mathbf{F}_{p} \mathfrak{g}$ be a homomorphism induced from the canonical map $G \rightarrow \mathfrak{g}$. Let $J^{\prime}$ be the kernel of this homomorphism. $J^{\prime}$ is generated by $\left\{g-1: g \in G_{p}\right\}$ over $\mathbf{F}_{p}$. Since $\mathbf{F}_{p}$ is a local commutative ring, we are able to apply Proposition 5.26 in Curtis-Reiner [2] to this homomorphism. We have $J^{\prime} \subset J . J / J^{\prime}$ is contained in the Jacobson radical of $\mathbf{F}_{p} G / J^{\prime}=\mathbf{F}_{p} \mathfrak{g}$. Therefore, $J / J^{\prime}=0$. We obtain $J=J^{\prime}$, and hence $\mathbf{F}_{p} G / J \cong \mathbf{F}_{p} \mathfrak{g}$.

We identify $\mathbf{F}_{p} G / J$ with $\mathbf{F}_{p} \mathfrak{g}$ in the remainder part. Put $f_{\Phi}=\operatorname{dim} \Delta_{\Phi}$. $f_{\Phi}$ equals $\operatorname{dim} \operatorname{Hom}_{R}\left(L_{\Phi}, L_{\Phi}\right)$, (c.f. Theorem 1.3.5, Benson [1]). We have

$$
\operatorname{dim} L_{\Phi}=f_{\Phi} d_{\Phi}
$$

Denote by $A$ the group ring $\mathbf{Z}_{p} \mathfrak{g}$. We see $R=A \otimes \mathbf{F}_{p}$. Since $p \nmid(\mathfrak{g}: 1)$, the center $Z(A)$ (resp. $Z(R))$ of $A$ (resp. $R$ ) is generated by $\{C(\sigma)=$ 
$\left.\frac{1}{(\mathfrak{g}: 1)} \sum_{\tau \in \mathfrak{g}} \tau \sigma \tau^{-1}: \sigma \in \mathfrak{g}\right\}$. Hence, $Z(R)$ is the image of $Z(A)$ with the canonical map. We have the assumption of (iii) of Theorem 1.9.4, Benson [1] is satisfied for $A$ and $R$. Thus, every $1_{\Phi}$ is lifted on a primitive central idempotent $\tilde{1}_{\Phi}$ of $A$ and

$$
1=\sum_{\Phi} \tilde{1}_{\Phi}
$$

Denote by $R_{\Phi}$ the simple ring $1_{\Phi} R$. Let $Y$ be a finitely generated $A$-module. We have an isomorphism $1_{\Phi}\left(Y \otimes \mathbf{F}_{p}\right) \cong \tilde{1}_{\Phi} Y \otimes \mathbf{F}_{p}$. Since $Y \otimes \mathbf{F}_{p} \cong R \otimes_{A} Y$, we see $\tilde{1}_{\Phi} Y \otimes \mathbf{F}_{p} \cong R_{\Phi} \otimes_{A} Y$. Denote by $r_{\Phi}(Y)$ a non-negative integer such that

$$
\tilde{1}_{\Phi} Y \otimes \mathbf{F}_{p} \cong R_{\Phi} \otimes_{A} Y \cong L_{\Phi}^{r_{\Phi}(Y)} .
$$

We use $R_{\Phi} \otimes_{A} Y$ rather than $\tilde{1}_{\Phi} Y \otimes \mathbf{F}_{p}$ in the sequel. We have

$$
r_{\Phi}(Y)=\frac{1}{f_{\Phi} d_{\Phi}} \operatorname{dim} R_{\Phi} \otimes_{A} Y, \quad \operatorname{dim} Y \otimes \mathbf{F}_{p}=\sum_{\Phi} r_{\Phi}(Y) f_{\Phi} d_{\Phi}
$$

Lemma 2. Let $0 \rightarrow X \rightarrow Y \rightarrow Z \rightarrow 0$ be an exact sequence of $A$ modules. Then, we have an exact sequence

$$
\operatorname{Tor}_{1}^{\mathbf{z}_{p}}\left(\tilde{1}_{\Phi} Z, \mathbf{F}_{p}\right) \rightarrow R_{\Phi} \otimes_{A} X \stackrel{f}{\rightarrow} R_{\Phi} \otimes_{A} Y \rightarrow R_{\Phi} \otimes_{A} Z \rightarrow 0 .
$$

If $\tilde{1}_{\Phi} Z$ is $\mathbf{Z}_{p}$-torsion free or if $\tilde{1}_{\Phi} Y$ is an $R$-module, $f$ is injective.

Proof. Let $U$ be an $A$-module. We have

$$
\operatorname{Tor}_{0}^{\mathbf{z}_{p}}\left(\tilde{1}_{\Phi} U, \mathbf{F}_{p}\right)=\tilde{1}_{\Phi} U \otimes \mathbf{z}_{p} \mathbf{F}_{p} \cong R_{\Phi} \otimes_{A} U .
$$

The exact sequence follows from an exact sequence

$$
0 \rightarrow \tilde{1}_{\Phi} X \rightarrow \tilde{1}_{\Phi} Y \rightarrow \tilde{1}_{\Phi} Z \rightarrow 0 .
$$

If $\tilde{1}_{\Phi} Z$ is $\mathbf{Z}_{p}$-torsion free, we have $\operatorname{Tor}_{1}^{\mathbf{Z}_{p}}\left(\tilde{1}_{\Phi} Z, \mathbf{F}_{p}\right)=0$. If $\tilde{1}_{\Phi} Y$ is an $R$ module, we have $\tilde{1}_{\Phi} Y=\tilde{1}_{\Phi} X \oplus \tilde{1}_{\Phi} Z$, because $R$ is semisimple. Hence, this short exact sequence splits. The lemma is proved.

By Lemma 1, we have

$$
1_{\Phi}\left(\mathcal{C} \otimes \mathbf{F}_{p} / J \mathcal{C} \otimes \mathbf{F}_{p}\right)=R_{\Phi} \otimes_{A}\left(\mathcal{C} / \mathcal{C}^{p} \cup \cup_{\sigma \in G_{p}} \mathcal{C}^{\sigma-1}\right) \cong 1_{\Phi} \operatorname{Gal}\left(H^{\text {cent }} / M\right) \otimes \mathbf{F}_{p}
$$

Let $C_{M}$ be the idele class group of $M$. By cup product with the canonical class of $H^{2}\left(G_{p}, C_{M}\right)$, an isomorphism $H^{-3}\left(G_{p}, \mathbf{Z}\right) \rightarrow H^{-1}\left(G_{p}, C_{M}\right)$ is 
defined. This isomorphism is a $\mathfrak{g}$-isomorphism. Furthermore, there is a surjective homomorphism onto $\operatorname{Gal}\left(H^{\text {cent }} / H^{\mathrm{ab}} M\right)$ :

$$
H^{-3}\left(G_{p}, \mathbf{Z}\right) \stackrel{\cong}{\longrightarrow} H^{-1}\left(G_{p}, C_{M}\right) \quad \longrightarrow \quad \mathrm{Gal}\left(H^{\text {cent }} / H^{\mathrm{ab}} M\right)
$$

(c.f. Jehne [4], Miyake [7], Shirai [8]). Since the $p$-primary torsion subgroup of $H^{-3}\left(G_{p}, \mathbf{Z}\right)$ is isomorphic to $H^{-3}\left(G_{p}, \mathbf{Z}_{p}\right), \tilde{1}_{\Phi} \operatorname{Gal}\left(H^{\text {cent }} / H^{\text {ab }} M\right)$ is a homomorphic image of $\tilde{1}_{\Phi} H^{-3}\left(G_{p}, \mathbf{Z}_{p}\right)$. By towers of Galois extensions $H^{\text {cent }} \supset H^{\mathrm{ab}} M \supset M$ and $H^{\mathrm{ab}} \supset M^{\mathrm{ab}} \supset K$, we have exact sequences

$$
\begin{gathered}
1 \rightarrow \operatorname{Gal}\left(H^{\mathrm{cent}} / H^{\mathrm{ab}} M\right) \rightarrow \operatorname{Gal}\left(H^{\mathrm{cent}} / M\right) \rightarrow \operatorname{Gal}\left(H^{\mathrm{ab}} / M^{\mathrm{ab}}\right) \rightarrow 1 \\
1 \rightarrow \operatorname{Gal}\left(H^{\mathrm{ab}} / M^{\mathrm{ab}}\right) \rightarrow \operatorname{Gal}\left(H^{\mathrm{ab}} / K\right) \rightarrow \operatorname{Gal}\left(M^{\mathrm{ab}} / K\right) \rightarrow 1
\end{gathered}
$$

Let $\Theta$ be the image of $H^{-3}\left(G_{p}, \mathbf{Z}_{p}\right)$ into $\operatorname{Gal}\left(H^{\text {cent }} / M\right) \otimes \mathbf{F}_{p}$ of the homomorphism obtained by combining the surjection of $H^{-3}\left(G_{p}, \mathbf{Z}_{p}\right)$ onto $\operatorname{Gal}\left(H^{\text {cent }} / H^{\mathrm{ab}} M\right)$ with the canonical map $\operatorname{Gal}\left(H^{\text {cent }} / H^{\mathrm{ab}} M\right) \otimes \mathbf{F}_{p} \rightarrow$ $\operatorname{Gal}\left(H^{\text {cent }} / M\right) \otimes \mathbf{F}_{p} \cdot \operatorname{Gal}\left(H^{\mathrm{ab}} / K\right)$ is an abelian $p$-group, because $\operatorname{Gal}\left(M^{\mathrm{ab}} / K\right)$ is an abelian $p$-group.

TheOREM 3. Denote by $\theta(\Phi)$ the value of $r_{\Phi}(\Theta)$. We have

$\theta(\Phi) \leq \frac{1}{f_{\Phi} d_{\Phi}} \operatorname{dim} \tilde{1}_{\Phi} H^{-3}\left(G_{p}, \mathbf{Z}_{p}\right) \otimes \mathbf{F}_{p}=\frac{1}{f_{\Phi} d_{\Phi}} \operatorname{dim} R_{\Phi} \otimes_{A} H^{-3}\left(G_{p}, \mathbf{Z}_{p}\right)$

Further, the value $a_{\Phi}=r_{\Phi}\left(\mathcal{C} \otimes \mathbf{F}_{p} / J \mathcal{C} \otimes \mathbf{F}_{p}\right)$ satisfies an inequality

$$
\begin{aligned}
r_{\Phi}\left(\operatorname{Gal}\left(H^{\mathrm{ab}} / M^{\mathrm{ab}}\right)\right)+\theta(\Phi)=a_{\Phi} \geq r_{\Phi}\left(\operatorname{Gal}\left(H^{\mathrm{ab}} / K\right)\right) & \\
& -r_{\Phi}\left(\operatorname{Gal}\left(M^{\mathrm{ab}} / K\right)\right)+\theta(\Phi) .
\end{aligned}
$$

If one of $\tilde{1}_{\Phi} \operatorname{Gal}\left(M^{\mathrm{ab}} / K\right), \tilde{1}_{\Phi} \operatorname{Gal}\left(H^{\mathrm{ab}} / K\right)^{p}$ and $\tilde{1}_{\Phi} \operatorname{Gal}\left(H^{\mathrm{ab}} / M^{\mathrm{ab}}\right)$ vanishes, we have

$$
\begin{gathered}
r_{\Phi}\left(\operatorname{Gal}\left(H^{\mathrm{ab}} / K\right)\right)=r_{\Phi}\left(\operatorname{Gal}\left(M^{\mathrm{ab}} / K\right)\right)+r_{\Phi}\left(\operatorname{Gal}\left(H^{\mathrm{ab}} / M^{\mathrm{ab}}\right)\right), \\
a_{\Phi}=r_{\Phi}\left(\operatorname{Gal}\left(H^{\mathrm{ab}} / K\right)\right)-r_{\Phi}\left(\operatorname{Gal}\left(M^{\mathrm{ab}} / K\right)\right)+\theta(\Phi) .
\end{gathered}
$$

Proof. Since $H^{-3}\left(G_{p}, \mathbf{Z}_{p}\right)$ is an $A$-module, we have

$$
r_{\Phi}\left(H^{-3}\left(G_{p}, \mathbf{Z}_{p}\right)\right)=\frac{1}{f_{\Phi} d_{\Phi}} \operatorname{dim} R_{\Phi} \otimes_{A} H^{-3}\left(G_{p}, \mathbf{Z}_{p}\right)
$$


from (1.2). Since $\Theta$ is a homomorphic image of $H^{-3}\left(G_{p}, \mathbf{Z}_{p}\right)$, we see $\theta(\Phi) \leq$ $r_{\Phi}\left(H^{-3}\left(G_{p}, \mathbf{Z}_{p}\right)\right)$. By applying Lemma 2 to the sequence (1.3), we have the following exact sequences:

$$
\begin{gathered}
0 \rightarrow R_{\Phi} \otimes_{A} \Theta \rightarrow R_{\Phi} \otimes_{A} \operatorname{Gal}\left(H^{\mathrm{cent}} / M\right) \rightarrow R_{\Phi} \otimes_{A} \operatorname{Gal}\left(H^{\mathrm{ab}} / M^{\mathrm{ab}}\right) \rightarrow 0 \\
\rightarrow R_{\Phi} \otimes_{A} \operatorname{Gal}\left(H^{\mathrm{ab}} / M^{\mathrm{ab}}\right) \stackrel{f}{\rightarrow} R_{\Phi} \otimes_{A} \operatorname{Gal}\left(H^{\mathrm{ab}} / K\right) \\
\rightarrow R_{\Phi} \otimes_{A} \operatorname{Gal}\left(M^{\mathrm{ab}} / K\right) \rightarrow 0
\end{gathered}
$$

The inequality concerning $a_{\Phi}$ follows from these sequences. We also have $f$ is injective if $\tilde{1}_{\Phi} \operatorname{Gal}\left(M^{\mathrm{ab}} / K\right)=0$ or if $\tilde{1}_{\Phi} \operatorname{Gal}\left(H^{\mathrm{ab}} / K\right)$ is an $R$-module, or if $\tilde{1}_{\Phi} \operatorname{Gal}\left(H^{\mathrm{ab}} / M^{\mathrm{ab}}\right)=0$. $\tilde{1}_{\Phi} \operatorname{Gal}\left(H^{\mathrm{ab}} / K\right)$ is an $R$-module if $\tilde{1}_{\Phi} \operatorname{Gal}\left(H^{\mathrm{ab}} / K\right)^{p}$ $=0$. If one of these conditions is satisfied, $r_{\Phi}\left(\mathrm{Gal}\left(H^{\mathrm{ab}} / M^{\mathrm{ab}}\right)\right)$ is equal to difference of $r_{\Phi}\left(\operatorname{Gal}\left(H^{\mathrm{ab}} / K\right)\right)$ and $r_{\Phi}\left(\operatorname{Gal}\left(M^{\mathrm{ab}} / K\right)\right)$. This proves the theorem.

Let $\mathfrak{h}$ be a normal subgroup of $\mathfrak{g}$ and put $\mathfrak{g}^{\prime}=\mathfrak{g} / \mathfrak{h}$. Denote by $R^{\prime}$ the group ring $\mathbf{F}_{p} \mathfrak{g}^{\prime}$. Let $I_{\mathfrak{h}}$ be the ideal of $R$ generated by $\{\sigma-1: \sigma \in \mathfrak{h}\}$. We have $R^{\prime} \cong R / I_{\mathfrak{h}}$. Denote by $\pi$ the canonical homomorphism $R \rightarrow R^{\prime}$. Note $\operatorname{im} \pi=R^{\prime}$ and ker $\pi=I_{\mathfrak{h}}$. Since $\mathfrak{h}$ is normal, an element

$$
1_{\mathfrak{h}}=\frac{1}{(\mathfrak{h}: 1)} \sum_{\sigma \in \mathfrak{h}} \sigma
$$

of $R$ is a central idempotent and ker $\pi \ni 1-1_{\mathfrak{h}}$. Denote by $\tilde{\pi}$ restriction of $\pi$ onto $1_{\mathfrak{h}} R$. $\tilde{\pi}$ is an isomorphism, because of $\pi\left(1_{\mathfrak{h}} R\right)=\pi(R)$ and $\operatorname{dim} 1_{\mathfrak{h}} R=$ $\operatorname{dim} R^{\prime}$. We have a decomposition

$$
R=1_{\mathfrak{h}} R \oplus\left(1-1_{\mathfrak{h}}\right) R, \quad 1_{\mathfrak{h}} R \cong R^{\prime}, \quad\left(1-1_{\mathfrak{h}}\right) R=I_{\mathfrak{h}} .
$$

Hence, ker $\pi=\left(1-1_{\mathfrak{h}}\right) R$. Let $\Psi$ be an irreducible character of $\mathfrak{g}^{\prime}$ and denote by $1_{\Psi}$ the corresponding primitive central idempotent of $R^{\prime}$. We see $\tilde{\pi}^{-1}\left(1_{\Psi}\right)$ is also a primitive central idempotent. Let $\Phi$ be an irreducible character afforded with a minimal left ideal of $\tilde{\pi}^{-1}\left(1_{\Psi}\right) R$. We have $1_{\Phi}=\tilde{\pi}^{-1}\left(1_{\Psi}\right)$ and $\Phi=\Psi \circ \pi$. Conversely, if $\sigma 1_{\Phi}=1_{\Phi}$ holds for every $\sigma \in \mathfrak{h}$, we have $1_{\Phi} 1_{\mathfrak{h}}=1_{\Phi}$. Hence, $1_{\Phi} R \cong 1_{\Phi} R^{\prime}$ by $(1.4)$. Let $\Psi$ be the character of $\mathfrak{g}^{\prime}$ afforded with a minimal left ideal of $1_{\Phi} R^{\prime}$. We have $\Phi=\Psi \circ \pi$. Therefore, we denote by the same symbol $\Phi$ this character $\Psi$.

LEMMA 4. Suppose there are normal subfields $K^{\prime}$ of $K / k$ and $M^{\prime}$ of $M / k$ such that $M^{\prime} \supset K^{\prime}, M=M^{\prime} K$ and $M^{\prime} \cap K=K^{\prime}$. Put $\mathfrak{h}=\operatorname{Gal}\left(K / K^{\prime}\right)$. 
Let $H^{\prime}$ be the $p$-Hilbert class field of $M^{\prime}$. Denote by $H^{\prime c e n t}$ the central $p$-genus field of $M^{\prime} / K^{\prime}$ and by $H^{\prime a b}$ the maximal abelian subfield of $H^{\prime} / K^{\prime}$. Let $\Theta^{\prime}$ be the image of $H^{-3}\left(G_{p}, \mathbf{Z}_{p}\right)$ into $\operatorname{Gal}\left(H^{\text {cent }} / M^{\prime}\right) \otimes \mathbf{F}_{p}$. Suppose $\sigma 1_{\Phi}=1_{\Phi}$ holds for every $\sigma \in \mathfrak{h}$. Then,

$$
r_{\Phi}\left(\operatorname{Gal}\left(H^{\mathrm{ab}} / K\right)\right)=r_{\Phi}\left(\operatorname{Gal}\left(H^{\prime \mathrm{ab}} / K^{\prime}\right)\right), \quad r_{\Phi}(\Theta)=r_{\Phi}\left(\Theta^{\prime}\right)
$$

Moreover, we have $r_{\Phi}\left(\operatorname{Gal}\left(H^{\text {cent }} / M\right)\right)=r_{\Phi}\left(\operatorname{Gal}\left(H^{\prime \text { cent }} / M^{\prime}\right)\right)$.

Proof. We have $\operatorname{Gal}\left(M^{\prime} / K^{\prime}\right) \cong G_{p}$ and $\operatorname{Gal}\left(M / M^{\prime}\right) \cong \mathfrak{h}$. Let $H_{1}$ be the maximal abelian subfield of $H / K^{\prime}$. We see $H^{\text {ab }} \supset H_{1} \cdot \operatorname{Gal}\left(H^{\mathrm{ab}} / K^{\prime}\right)$ is a semidirect product of the $p$-Sylow subgroup $\operatorname{Gal}\left(H^{\mathrm{ab}} / K\right)$ and $\mathfrak{h}$, because $K / K^{\prime}$ is a Galois extension and $p \chi\left[K: K^{\prime}\right]$. Hence, $\operatorname{Gal}\left(H_{1} K / K\right) \cong$ $\operatorname{Gal}\left(H^{\mathrm{ab}} / K\right) / \prod_{\sigma \in \mathfrak{h}} \operatorname{Gal}\left(H^{\mathrm{ab}} / K\right)^{\sigma-1}$. We have the following isomorphism of $R$-modules:

$$
\operatorname{Gal}\left(H_{1} K / K\right) \otimes \mathbf{F}_{p} \cong \operatorname{Gal}\left(H^{\mathrm{ab}} / K\right) \otimes \mathbf{F}_{p} / \sum_{\sigma \in \mathfrak{h}}\left(\operatorname{Gal}\left(H^{\mathrm{ab}} / K\right) \otimes \mathbf{F}_{p}\right)^{\sigma-1}
$$

$\operatorname{By}(1.4)$, we see $\operatorname{Gal}\left(H^{\mathrm{ab}} / K\right) \otimes \mathbf{F}_{p}=1_{\mathfrak{h}} \operatorname{Gal}\left(H^{\mathrm{ab}} / K\right) \otimes \mathbf{F}_{p} \oplus I_{\mathfrak{h}} \operatorname{Gal}\left(H^{\mathrm{ab}} / K\right) \otimes$ $\mathbf{F}_{p}$. Thus, we have $1_{\Phi} \operatorname{Gal}\left(H_{1} K / K\right) \otimes \mathbf{F}_{p} \cong 1_{\Phi} \operatorname{Gal}\left(H^{\mathrm{ab}} / K\right) \otimes \mathbf{F}_{p}$. Namely,

$$
r_{\Phi}\left(\operatorname{Gal}\left(H_{1} K / K\right)\right)=r_{\Phi}\left(\operatorname{Gal}\left(H^{\mathrm{ab}} / K\right)\right) .
$$

Let $H_{2}$ be the intermediate field corresponding to $\prod_{\sigma \in \mathfrak{h}} \mathcal{C}^{\sigma-1}$ in $H / M$. Since $\mathfrak{h}$ acts on $\operatorname{Gal}\left(H_{1} M / M\right)$ trivially, we have $H_{2} \supset H_{1}$. Moreover, since $p \backslash \sharp \sharp H^{-3}(\mathfrak{h}, \mathbf{Z})$, the central $p$-genus field of $M / M^{\prime}$ coincides with the abelian $p$-genus field of $M / M^{\prime}$. We have $H_{2}=H^{\prime} M$. Note $H^{\prime} M=H^{\prime} K$, because of $H^{\prime} \supset M^{\prime}$ and $M=M^{\prime} K$. Let $H_{2}^{\text {ab }}$ be the maximal abelian subfield of $H_{2} / K$. Since $H_{2}=H^{\prime} K$, we have $\operatorname{Gal}\left(H_{2} / K\right) \cong \operatorname{Gal}\left(H^{\prime} / K^{\prime}\right)$. Taking the maximal abelian quotients, we obtain $H_{2}^{\mathrm{ab}}=H^{\prime} \mathrm{ab} K$. This implies $H_{1} K=H^{\prime \mathrm{ab}} K$, because of $H_{2}^{\mathrm{ab}} \supset H_{1} \supset H^{\prime \mathrm{ab}}$. Therefore, we have $\operatorname{Gal}\left(H_{1} K / K\right) \cong \operatorname{Gal}\left(H^{\prime a b} / K^{\prime}\right)$ and

$$
r_{\Phi}\left(\operatorname{Gal}\left(H_{1} K / K\right)\right)=r_{\Phi}\left(\operatorname{Gal}\left(H^{\prime a b} / K^{\prime}\right)\right) .
$$

This proves $r_{\Phi}\left(\operatorname{Gal}\left(H^{\mathrm{ab}} / K\right)\right)=r_{\Phi}\left(\operatorname{Gal}\left(H^{\prime \mathrm{ab}} / K^{\prime}\right)\right)$.

Let $N_{M / M^{\prime}}$ be the norm map. By extending ideals of $M^{\prime}$ onto $M$, we have a natural map $j_{M / M^{\prime}}: \mathcal{C}^{\prime} \rightarrow \mathcal{C}$. Since $N_{M / M^{\prime}} \circ j_{M / M^{\prime}}$ is $\left[M: M^{\prime}\right]$-th power map, $j_{M / M^{\prime}}$ is injective and $N_{M / M^{\prime}}$ is surjective. Note

$$
\frac{1}{(\mathfrak{h}: 1)} j_{M / M^{\prime}} \circ N_{M / M^{\prime}}=\tilde{1}_{\mathfrak{h}} \text {. }
$$


We have $\tilde{1}_{\mathfrak{h}} \mathcal{C} \cong \mathcal{C}^{\prime}$. Hence, $1_{\mathfrak{h}} \mathcal{C} \otimes \mathbf{F}_{p} \cong \mathcal{C}^{\prime} \otimes \mathbf{F}_{p}$. Further, since $1_{\mathfrak{h}} \sigma=\sigma 1_{\mathfrak{h}}$ for every $\sigma \in G_{p}$, we have $\tilde{1}_{\mathfrak{h}} J \mathcal{C} \otimes \mathbf{F}_{p} \cong J \tilde{1}_{\mathfrak{h}} \mathcal{C} \otimes \mathbf{F}_{p}$. Therefore,

$$
1_{\mathfrak{h}}\left(\mathcal{C} \otimes \mathbf{F}_{p} / J \mathcal{C} \otimes \mathbf{F}_{p}\right) \cong \mathcal{C}^{\prime} \otimes \mathbf{F}_{p} / J \mathcal{C}^{\prime} \otimes \mathbf{F}_{p}
$$

This proves $r_{\Phi}\left(\mathcal{C} \otimes \mathbf{F}_{p} / J \mathcal{C} \otimes \mathbf{F}_{p}\right) \cong r_{\Phi}\left(\mathcal{C}^{\prime} \otimes \mathbf{F}_{p} / J \mathcal{C}^{\prime} \otimes \mathbf{F}_{p}\right)$.

We have a commutative diagram

$$
\begin{aligned}
& \tilde{1}_{\Phi} \operatorname{Gal}\left(H^{\mathrm{cent}} / M\right) \otimes \mathbf{F}_{p} \\
& \tilde{1}_{\Phi} H^{-3}\left(G_{p}, \mathbf{z}_{p}\right) \\
& \tilde{1}_{\Phi} \operatorname{Gal}\left(H^{\prime \text { cent }} / M^{\prime}\right) \otimes \mathbf{F}_{p}
\end{aligned}
$$

Thus, $1_{\Phi} \Theta \cong 1_{\Phi} \Theta^{\prime}$. The lemma is proved.

\section{$\S 2$. The Kummer group}

We denote by $\zeta_{m}$ a primitive $m$-th root of unity. We are able to calculate the value of $r_{\Phi}(\mathcal{C})$ in $M\left(\zeta_{p}\right)$ by virtue of Lemma 4 if $M \not \supset \zeta_{p}$. We may suppose $\zeta_{p} \in M$. Let $H^{*}$ be the maximal elementary $(p, \cdots, p)$-abelian subfield of $H / K$. We see $\operatorname{Gal}\left(H^{*} / K\right)=\operatorname{Gal}\left(H^{\mathrm{ab}} / K\right) \otimes \mathbf{F}_{p}$. Let $B=H^{\times p} \cap$ $K^{\times} / K^{\times p}$ be the Kummer group of $H^{*} / K$. We consider $B$ a submodule of $K^{\times} \otimes \mathbf{F}_{p}=K^{\times} / K^{\times p}$. Denote by $\mathbf{T}$ an $R$-module $\left\langle\zeta_{p}>\otimes \mathbf{F}_{p}\right.$. The Kummer pairing is a non-degenerate pairing having values in $\mathbf{T}$ :

$$
<b, g>=\sqrt[p]{b^{g-1}} \otimes 1, \quad b \in B, \quad g \in \operatorname{Gal}\left(H^{*} / K\right) .
$$

Since $H^{*}$ is Galois over $k, B$ and $\operatorname{Gal}\left(H^{*} / K\right)$ are $R$-modules. Let $\omega$ be an irreducible character afforded with $\mathbf{T}$. The action of $\sigma \in \mathfrak{g}$ satisfies the following relation on the pairing

$$
<\sigma b, \sigma g>=\omega(\sigma)<b, g>\text {. }
$$

Let $\hat{\Phi}$ be a character defined by $\hat{\Phi}(\sigma)=\Phi\left(\sigma^{-1}\right)$. The reflection of $\Phi$ is defined to be a character $\hat{\Phi} \omega$. Denote by $\Phi^{*}$ the reflection of $\Phi$. Let $\hat{L}_{\Phi}=$ $\operatorname{Hom}\left(L_{\Phi}, \mathbf{F}_{p}\right)$ be an $R$-module where $\sigma \in \mathfrak{g}$ acts by $\sigma f=f \circ \sigma^{-1}$ for $f \in \hat{L}_{\Phi}$. Let $L_{\Phi}^{*}=\hat{L}_{\Phi} \otimes \mathbf{T}$ be an $R$-module where $\mathfrak{g}$ acts diagonally. $\hat{\Phi}$ (resp. $\Phi^{*}$ ) is afforded with $\hat{L}_{\Phi}\left(\operatorname{resp} . L_{\Phi}^{*}\right)$.

Lemma 5. We have 
(1) $\hat{\hat{L}}_{\Phi} \cong L_{\Phi}$ and $L_{\Phi}^{* *} \cong L_{\Phi}$

(2) $\Phi^{*}$ is irreducible,

(3) $f_{\Phi}=f_{\Phi^{*}}$ and $d_{\Phi}=d_{\Phi^{*}}$.

Proof. By (iii) of Lemma 10.26, Curtis-Reiner [2], we have $\hat{\hat{L}}_{\Phi} \cong$ $L_{\Phi}$. When finitely generated $R$-modules $L_{1}$ and $L_{2}$ are given, we consider $\operatorname{Hom}\left(L_{1}, L_{2}\right)$ (resp. $L_{1} \otimes L_{2}$ ) be an $R$-module by $\sigma f=\sigma \circ f \circ \sigma^{-1}$ (resp. $\sigma(x \otimes y)=\sigma x \otimes \sigma y)$ for $f \in \operatorname{Hom}\left(L_{1}, L_{2}\right)$ (resp. $\left.x \otimes y \in L_{1} \otimes L_{2}\right)$. By Proposition 10.30, Curtis-Reiner [2], we have

$$
\hat{L}_{1} \otimes L_{2} \cong \operatorname{Hom}\left(L_{1}, L_{2}\right),
$$

where $\hat{L}_{1}=\operatorname{Hom}\left(L_{1}, \mathbf{F}_{p}\right)$. Since $L_{\Phi}^{* *} \cong \operatorname{Hom}\left(L_{\Phi}^{*}, \mathbf{F}_{p}\right) \otimes \mathbf{T}$ and $\hat{\mathbf{T}} \otimes \mathbf{T} \cong \mathbf{F}_{p}$, an isomorphism $L_{\Phi}^{* *} \cong L_{\Phi}$ follows from

$$
\begin{aligned}
\operatorname{Hom}\left(\hat{L}_{\Phi} \otimes \mathbf{T}, \mathbf{F}_{p}\right) \otimes \mathbf{T} & \cong \operatorname{Hom}\left(\mathbf{T}, \operatorname{Hom}\left(\hat{L}_{\Phi}, \mathbf{F}_{p}\right)\right) \otimes \mathbf{T} \\
& \cong \operatorname{Hom}\left(\mathbf{T}, L_{\Phi}\right) \otimes \mathbf{T} \cong \hat{\mathbf{T}} \otimes L_{\Phi} \otimes \mathbf{T} \cong L_{\Phi}
\end{aligned}
$$

Suppose $L_{\Phi}^{*}=M_{1} \oplus M_{2}$ for non-trivial submodules $M_{i}$. We have $L_{\Phi}^{* *} \cong$ $M_{1}^{*} \oplus M_{2}^{*}$. However, this contradicts to that $L_{\Phi}$ is simple. Thus, $L_{\Phi}^{*}$ is simple and $\Phi^{*}$ is irreducible. Since $\operatorname{Hom}\left(L_{\Phi}, L_{\Phi}\right)^{\mathfrak{g}}=\operatorname{Hom}_{R}\left(L_{\Phi}, L_{\Phi}\right)$ and $\hat{L}_{\Phi} \otimes L_{\Phi} \cong$ $\operatorname{Hom}\left(L_{\Phi}, L_{\Phi}\right)$, we have $\left(\hat{L}_{\Phi} \otimes L_{\Phi}\right)^{\mathfrak{g}} \cong \operatorname{Hom}_{R}\left(L_{\Phi}, L_{\Phi}\right)$. By (10.32), CurtisReiner [2], we also have $\operatorname{Hom}_{R}\left(L_{\Phi}, L_{\Phi}\right) \cong \operatorname{Hom}_{R}\left(\hat{L}_{\Phi}, \hat{L}_{\Phi}\right)$. This implies $\left(\hat{L}_{\Phi} \otimes L_{\Phi}\right)^{\mathfrak{g}} \cong\left(L_{\Phi} \otimes \hat{L}_{\Phi}\right)^{\mathfrak{g}}$. Since $\hat{L}_{\Phi^{*}} \otimes L_{\Phi^{*}}=\operatorname{Hom}\left(\hat{L}_{\Phi} \otimes \mathbf{T}, \mathbf{F}_{p}\right) \otimes\left(\hat{L}_{\Phi} \otimes \mathbf{T}\right) \cong$ $\left(\hat{\mathbf{T}} \otimes L_{\Phi}\right) \otimes\left(\hat{L}_{\Phi} \otimes \mathbf{T}\right) \cong L_{\Phi} \otimes \hat{L}_{\Phi}$, we obtain $\left(\hat{L}_{\Phi^{*}} \otimes L_{\Phi^{*}}\right) \mathfrak{g} \cong\left(L_{\Phi} \otimes \hat{L}_{\Phi}\right)^{\mathfrak{g}}$. Therefore,

$$
\begin{aligned}
& f_{\Phi}=\operatorname{dim} \operatorname{Hom}_{R}\left(L_{\Phi}, L_{\Phi}\right)=\operatorname{dim}\left(\hat{L}_{\Phi} \otimes L_{\Phi}\right)^{\mathfrak{g}} \\
& \quad=\operatorname{dim}\left(\hat{L}_{\Phi^{*}} \otimes L_{\Phi^{*}}\right)^{\mathfrak{g}}=\operatorname{dim} \operatorname{Hom}_{R}\left(L_{\Phi^{*}}, L_{\Phi^{*}}\right)=f_{\Phi^{*}} .
\end{aligned}
$$

Since $\operatorname{dim} L_{\Phi}=\operatorname{dim} L_{\Phi^{*}}$, we have $d_{\Phi}=d_{\Phi^{*}}$ from (1.1).

We have $1_{\Phi} B$ and $1_{\Phi^{*}} \operatorname{Gal}\left(H^{*} / K\right)$ are dual to each other by means of the Kummer pairing. Hence, a formula

$$
\operatorname{dim} 1_{\Phi} B=\operatorname{dim} 1_{\Phi^{*}} \operatorname{Gal}\left(H^{*} / K\right)
$$

is obtained. Since $f_{\Phi} d_{\Phi}=f_{\Phi^{*}} d_{\Phi^{*}}$, we have

$$
r_{\Phi}(B)=r_{\Phi^{*}}\left(\operatorname{Gal}\left(H^{\mathrm{ab}} / K\right)\right)
$$


from (1.2) and (2.1).

In accordance with Leopoldt [6], we are able to obtain a formula for $r_{\Phi}(B)$ by modifying the argument developed there. Denote by $K_{v}$ the completion of $K$ at a place $v$. Let $U_{v}$ be the unit group of $K_{v}$ when $v$ is a finite place. Let $\mu_{v}$ be the $p$-primary torsion subgroup of $K_{v}^{\times}$. We have a tower of Kummer extensions:

$$
K_{v}^{*}=K_{v}\left(\sqrt[p]{x}: x \in K_{v}^{\times}\right) \supset \tilde{K}_{v}^{*}=K_{v}\left(\sqrt[p]{x}: x \in U_{v}\right) \supset K_{v} .
$$

Denote by $v_{0}$ a place of $k$. Let $v$ (resp. $w$ ) denote a prolongation of $v_{0}$ (resp. $v$ ) onto $K$ (resp. $M)$. Suppose $v_{0} \mid p$. Let $p^{f}$ be the absolute degree of the valuation ideal $\mathfrak{p}_{v} \cdot K_{v}\left(\zeta_{p^{p f}-1}\right)$ is the unramified abelian extension of degree $p$ over $K_{v}$. Put

$$
\tilde{\xi}_{v}=\sum_{i=0}^{p-1} \zeta_{p^{p f-1}}^{p^{f i}} \zeta_{p}^{i} .
$$

We have $\tilde{\xi}_{v}^{p} \in K_{v}$ and $K_{v}\left(\zeta_{p^{p f}-1}\right)=K_{v}\left(\tilde{\xi}_{v}\right)$. Let $\xi_{v}$ be an element of $U_{v}$ such that $\tilde{\xi}_{v}^{p} \in \xi_{v} K_{v}^{\times p}$. We have $K_{v}\left(\sqrt[p]{\xi_{v}}\right)=K_{v}\left(\zeta_{p^{p f}-1}\right)$. Let $K_{v}^{\prime}$ be the inertia field in $M_{w} / K_{v}$. Similarly, there is an unit $\xi_{v}^{\prime}$ of $K_{v}^{\prime}$ such that $K_{v}^{\prime}\left(\sqrt[p]{\xi_{v}^{\prime}}\right)$ is the unramified abelian extension of degree $p$ over $K_{v}^{\prime}$.

LEMma 6. Let $v_{0}$ be a finite place of $k$. Let a be an element of $K_{v}^{\times}$.

(1) Suppose $v_{0} \not p . M_{w}(\sqrt[p]{a}) / M_{w}$ is unramified if and only if the ramification index of $K_{v}(\sqrt[p]{a}) / K_{v}$ divides that of $M_{w} / K_{v}$.

(2) Suppose $v_{0} \mid p$. Let $M_{w}^{\mathrm{ab}}$ be the maximal abelian subfield of $M_{w} / K_{v}$. We have $M_{w}(\sqrt[p]{a})$ is unramified if and only if $\sqrt[p]{a} \in M_{w}^{\mathrm{ab}}\left(\sqrt[p]{\xi_{v}^{\prime}}\right)$.

Proof. Since $M_{w}(\sqrt[p]{a}) / M_{w}$ is tamely ramified for $v_{0} \not p,(1)$ is obvious. $M_{w}\left(\sqrt[p]{\xi_{v}^{\prime}}\right)$ is unramified and of degree $p$ over $M_{w}$ for $v_{0} \mid p$. Let $G_{w}\left(\operatorname{resp} . G_{w}^{\prime}\right)$ be the Galois group of $\left.M_{w} / K_{v}\left(\operatorname{resp} . M_{w}\left(\sqrt[p]{\xi_{v}^{\prime}}\right) / K_{v}\right)\right)$. Denote by $G_{w}^{c}$ and $G_{w}^{\prime c}$ the subgroups generated by commutators. Since $G_{w}$ is a homomorphic image of $G_{w}^{\prime}$, we have $\sharp G_{w}^{c} \leq \sharp G_{w}^{\prime c}$. We see $\sharp G_{w}^{\prime}=p \sharp G_{w}$ and $\left(G_{w}^{\prime}: G_{w}^{\prime c}\right) \geq$ $p\left(G_{w}: G_{w}^{c}\right)$. Hence, $\sharp G_{w}^{c}=\sharp G_{w}^{c}$. This implies that the maximal abelian subfield of $M_{w}\left(\sqrt[p]{\xi_{v}^{\prime}}\right) / K_{v}$ is $M_{w}^{\mathrm{ab}}\left(\sqrt[p]{\xi_{v}^{\prime}}\right)$. This proves $(2)$, because $K_{v}(\sqrt[p]{a})$ is an abelian extension of $K_{v}$.

Let $P$ be the set consisting of every place of $k_{0}$ lying above $p$. Denote by $P(K)$ the set of every prolongation onto $K$ of every place contained in 
$P$. We use this convention for an arbitrary set of places of $k$. Denote by $e(w / v)$ the ramification index of $M_{w} / K_{v}$ for a finite place $v_{0}$. Let $T$ and $S$ be sets of places of $k$ defined by

$$
\begin{aligned}
T & =\left\{v_{0} \notin P: v_{0} \not \infty, p \mid e(w / v)\right\} \cup\left\{v_{0} \in P: M_{w}^{\mathrm{ab}}\left(\sqrt[p]{\xi_{v}^{\prime}}\right) \cap K_{v}^{*} \not \subset \tilde{K}_{v}^{*}\right\}, \\
S & =\left\{v_{0}: w \text { is real and } K_{v}^{\times p} \neq K_{v}^{\times}\right\}
\end{aligned}
$$

respectively. Let $V_{v}$ be a closed subgroup of $K_{v}^{\times}$such that

$$
V_{v}=\left\{\begin{array}{lll}
M_{w}^{\mathrm{ab}}\left(\sqrt[p]{\xi_{v}^{\prime}}\right)^{\times p} \cap U_{v} & \text { if } \quad v \in P(K) \backslash T(K), \\
M_{w}^{\mathrm{ab}}\left(\sqrt[p]{\xi_{v}^{\prime}}\right)^{\times p} \cap K_{v}^{\times} & \text {if } \quad v \in P(K) \cap T(K), \\
K_{v}^{\times p} & \text { if } \quad v \in S(K) .
\end{array}\right.
$$

Note $V_{v}$ contains $U_{v}^{p}$ (resp. $\left.K_{v}^{\times p}\right)$ if $v \in P(K) \backslash T(K)$ (resp. $v \in P(K) \cap$ $T(K))$. We observe that $M(\sqrt[p]{a}) / M$ is unramified for $a \in K^{\times}$if and only if $a \in V_{v} K_{v}^{\times p}$ for every $v \in P(K) \cup S(K)$ and $v(a) \equiv 0 \bmod p$ for every finite places $v$ not contained in $T(K)$, where we abuse notation and denote by the same symbol $v$ the normalized additive valuation belonging to $v$. $B$ is a subgroup of $K^{\times} \otimes \mathbf{F}_{p}$ consisting of $a \otimes 1$ for every $a \in K^{\times}$satisfying this condition.

Let $E_{T}$ be the group of $T$-units of $K: E_{T}=\left\{a \in K^{\times}: v(a)=0\right.$ for every finite place $v \notin T(K)\}$. Let $P_{T}=K^{\times} / E_{T}$ and $D_{T}=\oplus_{v \nmid \infty, v \notin T(K)} K_{v}^{\times} / U_{v}$. $P_{T}$ is considered a subgroup of $D_{T}$ by a diagonal map. We have an exact sequence

$$
0 \rightarrow E_{T} \otimes \mathbf{F}_{p} \stackrel{i}{\rightarrow} K^{\times} \otimes \mathbf{F}_{p} \stackrel{j}{\rightarrow} P_{T} \otimes \mathbf{F}_{p} \rightarrow 0
$$

because $P_{T}$ is torsion free. Put $B_{1}=i^{-1}(\operatorname{ker} j \cap B) . j(B)$ consists of $(a) \otimes 1$ such that $a \otimes 1 \in B$. Let $C_{T}$ be the $p$-torsion subgroup of $D_{T} / P_{T}$. Let $(a) \otimes 1$ be an element of $j(B)$ with $a \otimes 1 \in B$. Since $v(a) \equiv 0 \bmod p$ for every finite place $v \notin T(K)$, there is $\mathfrak{a} \in D_{T}$ such that $\mathfrak{a}^{p}=(a)$. We observe $\mathfrak{a}$ is principal if and only if $a \in K^{\times p} E_{T}$. Since $a \in K^{\times p} E_{T}$ implies $a \otimes 1 \in \operatorname{ker} j$, a correspondence $(a) \otimes 1 \rightarrow \operatorname{cl}(\mathfrak{a})$ defines an injective homomorphism of $j(B)$ into $C_{T}$. Denote by $B_{0}$ the image of this homomorphism. Observe that $\operatorname{cl}(\mathfrak{a}) \in B_{0}$ if and only if there is $a \in K^{\times}$such that $\mathfrak{a}^{p}=(a)$ and $a \in V_{v} K_{v}^{\times p}$ for every $v \in P(K) \cup S(K)$. We have Leopoldt's decomposition of the Kummer group $B$ :

$$
0 \rightarrow B_{1} \rightarrow B \rightarrow B_{0} \rightarrow 0
$$


c.f. Leopoldt [6]. Hence, we obtain a formula

$$
r_{\Phi}(B)=r_{\Phi}\left(B_{1}\right)+r_{\Phi}\left(B_{0}\right)
$$

Let $V$ be a direct product of $V_{v}$ for $v \in P(K) \cup S(K)$. Put

$$
U=\prod_{v \in P(K) \backslash T(K)} U_{v} \times \prod_{v \in P(K) \cap T(K)} K_{v}^{\times} \times \prod_{v \in S(K)} K_{v}^{\times} .
$$

$V$ is an open subgroup of $U$. Let $\iota: E_{T} \rightarrow U$ be the diagonal map. $\iota\left(E_{T}\right) V$ is also an open subgroup. Let $U^{\prime}$ be a closed subgroup of the idele group of $K$ such that

$$
U^{\prime}=\prod_{v \nmid \infty, v \notin P(K) \cup T(K)} U_{v} \times \prod_{v \in T(K) \backslash P(K)} K_{v}^{\times} \times \prod_{v \mid \infty, v \notin S(K)} K_{v}^{\times} .
$$

Let $K_{0}$ be the class field of $K$ corresponding to an open subgroup $K^{\times} U U^{\prime}$ of the idele group of $K$. Let $K_{1}$ be a class field of $K$ such that $K_{1} \supset K_{0}$ and $\operatorname{Gal}\left(K_{1} / K_{0}\right) \cong K^{\times} U U^{\prime} / K^{\times} V U^{\prime}$. Since $K^{\times} \cap U U^{\prime}=E_{T}$, we have $\operatorname{Gal}\left(K_{1} / K_{0}\right) \cong U U^{\prime} / E_{T} V U^{\prime}$. Further, by projection onto $U$, we have

$$
\operatorname{Gal}\left(K_{1} / K_{0}\right) \cong U / \iota\left(E_{T}\right) V
$$

Since $V \supset U^{p}$, we have a homomorphism $E_{T} \otimes \mathbf{F}_{p} \rightarrow U / V$. Observe that the cokernel is $U / \iota\left(E_{T}\right) V$ and that the kernel is $B_{1}$. We have an exact sequence

$$
0 \rightarrow B_{1} \rightarrow E_{T} \otimes \mathbf{F}_{p} \rightarrow U / V \rightarrow \operatorname{Gal}\left(K_{1} / K_{0}\right) \rightarrow 0
$$

Let $K_{P \backslash T}^{\times}$be a subgroup of $K^{\times}$consisting of $a$ such that $v(a)=0$ for every $v \in P(K) \backslash T(K)$. We extend $\iota$ onto $K_{P \backslash T}^{\times}$. Note $\iota\left(K_{P \backslash T}^{\times}\right)^{p} \subset V$. Each element $c \in C_{T}$ contains $\mathfrak{a} \in D_{T}$ such that $\mathfrak{a}^{p}$ is generated by an element $a$ of $K_{P \backslash T}^{\times}$. If there is $\mathfrak{a}^{\prime} \in c$ such that $\mathfrak{a}^{\prime p}=\left(a^{\prime}\right)$ for $a^{\prime} \in K_{P \backslash T}^{\times}$, we have $b \in K^{\times}$and $x \in E_{T}$ such that $a=x b^{p} a^{\prime}$. We see $\iota\left(b^{p}\right) \in V$, because $b^{p} \in K_{P \backslash T}^{\times}$means $b \in K_{P \backslash T}^{\times}$. Hence, $\iota(a) \iota\left(E_{T}\right) V=\iota\left(a^{\prime}\right) \iota\left(E_{T}\right) V$. Therefore, a homomorphism

$$
\rho: C_{T} \rightarrow \operatorname{Gal}\left(K_{1} / K_{0}\right)
$$

is well-defined by $\rho(c)=\iota\left(a E_{T}\right) V$. Let $(a) \otimes 1 \in j(B)$. We may assume $a \in V_{v} K_{v}^{\times p}$ for every $v \in P(K) \cup S(K)$. Note $V_{v} K_{v}^{\times p}=V_{v}$ for $v \in S(K)$. By approximation theorem of valuations, there exists $b_{v} \in K^{\times}$for each $v \in$ $P(K) \backslash T(K)$ such that $v\left(b_{v}\right)=1$ and $u\left(b_{v}\right)=0$ for every $u \in P(K) \backslash(T(K) \cup$ 
$\{v\})$. Since $v\left(a b_{v}^{-v(a)}\right)=0$ and $v(a) \equiv 0 \bmod p$, there is $b \in K^{\times}$such that $a b^{p} \in K_{P \backslash T}^{\times}$. We have $\iota\left(a b^{p}\right) \in V$. This implies $B_{0} \subset$ ker $\rho$. Conversely, let $c l(\mathfrak{a}) \in \operatorname{ker} \rho$. There are $a \in K_{P \backslash T}^{\times}, b \in K^{\times}$such that $(a)=\left(b^{p}\right) \mathfrak{a}^{p}$, and there are $x \in E_{T}, w \in V$ such that $\iota(a)=\iota(x) w$. Since $\iota\left(a x^{-1}\right) \in V$, we have $a x^{-1} \otimes 1 \in B$. This means $\operatorname{cl}(\mathfrak{a}) \in B_{0}$. We have ker $\rho \subset B_{0}$. Therefore, another exact sequence

$$
0 \rightarrow B_{0} \rightarrow C_{T} \stackrel{\rho}{\rightarrow} \operatorname{Gal}\left(K_{1} / K_{0}\right)
$$

is obtained. Let $\alpha(\Phi)=r_{\Phi}(\operatorname{coker} \rho)$

ThEOREM 7. Put $\beta_{T}(\Phi)=r_{\Phi}\left(E_{T} \otimes \mathbf{F}_{p}\right), \gamma_{T}(\Phi)=r_{\Phi}\left(C_{T}\right), \kappa_{1}(\Phi)=$ $r_{\Phi}\left(U \otimes \mathbf{F}_{p}\right)$ and $\kappa_{2}(\Phi)=r_{\Phi}\left(V / U^{p}\right)$. We have $r_{\Phi^{*}}\left(\operatorname{Gal}\left(H^{\mathrm{ab}} / K\right)\right)=r_{\Phi}\left(B_{1}\right)+$ $r_{\Phi}\left(B_{0}\right)$ and

$\beta_{T}(\Phi)+\gamma_{T}(\Phi) \geq r_{\Phi}\left(B_{1}\right)+r_{\Phi}\left(B_{0}\right)=\alpha(\Phi)+\beta_{T}(\Phi)+\gamma_{T}(\Phi)-\kappa_{1}(\Phi)+\kappa_{2}(\Phi)$.

Proof. By (2.2) and (2.4), the value of $r_{\Phi^{*}}\left(\operatorname{Gal}\left(H^{\mathrm{ab}} / K\right)\right)$ equals the sum of $r_{\Phi}\left(B_{1}\right)$ and $r_{\Phi}\left(B_{0}\right)$. We see $\beta_{T}(\Phi) \geq r_{\Phi}\left(B_{1}\right)$ and $\gamma_{T}(\Phi) \geq r_{\Phi}\left(B_{0}\right)$. Hence $\beta_{T}(\Phi)+\gamma_{T}(\Phi) \geq r_{\Phi^{*}}\left(\operatorname{Gal}\left(H^{\mathrm{ab}} / K\right)\right)$. Since $U / V \cong\left(U / U^{p}\right) /\left(V / U^{p}\right)$, we have $r_{\Phi}(U / V)=r_{\Phi}\left(U / U^{p}\right)-r_{\Phi}\left(V / U^{p}\right)$. The values of $r_{\Phi}\left(B_{1}\right)$ and $r_{\Phi}\left(B_{0}\right)$ are described with $\alpha(\Phi), \beta_{T}(\Phi), \gamma_{T}(\Phi)$ and $\kappa_{i}(\Phi)$ by means of the sequences (2.6) and (2.8).

\section{$\S 3$. The character of the representation on $\operatorname{Gal}\left(H^{*} / K\right)$}

The character afforded with a finitely generated $R$-module $Y$ may have no significance. We have

$$
Y=\bigoplus_{\Phi} 1_{\Phi} Y \cong L_{\Phi}^{r_{\Phi}(Y)}
$$

However, if $r_{\Phi}(Y) \equiv 0 \bmod p$ for every $\Phi$, the character is 0 . By this reason, we introduce a free abelian group $c h(R)$ on $\mathfrak{B}(\mathfrak{g}) \cdot \sum m_{\Phi} \Phi>\sum n_{\Phi} \Phi$ means $m_{\Phi} \geq n_{\Phi}$ holds for every $\Phi$ and there is at least one $\Phi$ such that $m_{\Phi}>n_{\Phi}$. In the sequel, if we say a character afforded with $Y$ or a character of the representation on $Y$, we mean an element $\sum_{\Phi} r_{\Phi}(Y) \Phi$ of $\operatorname{ch}(R)$.

Let $\alpha$ (resp. $\beta_{T}, \gamma_{T}$ ) be the character of the representation of $\mathfrak{g}$ on an $R$-module coker $\rho$ (resp. $\left.E_{T} \otimes \mathbf{F}_{p}, C_{T}\right)$. Let $\kappa_{1}$ (resp. $\left.\kappa_{2}\right)$ be the character afforded with an $R$-module $U \otimes \mathbf{F}_{p}\left(\right.$ resp. $\left.V / U^{p}\right)$. Let $\varphi_{B}$ be the character afforded with $B$. By virtue of Theorem 7 , we have

$$
\beta_{T}+\gamma_{T} \geq \varphi_{B}=\alpha+\beta_{T}+\gamma_{T}-\kappa_{1}+\kappa_{2}
$$


LEMma 8. Let $P$ and $Q$ be finitely generated $\mathbf{Z}_{p}$-torsion free $A$-modules. If $P \otimes \mathbf{z}_{p} \mathbf{Q}_{p} \cong Q \otimes \mathbf{z}_{p} \mathbf{Q}_{p}$, we have $P \otimes \mathbf{z}_{p} \mathbf{F}_{p} \cong Q \otimes \mathbf{z}_{p} \mathbf{F}_{p}$.

Proof. By virtue of Corollary 18.16, Curtis-Reiner [2], we have $P \cong Q$ if $P \otimes \mathbf{z}_{p} \mathbf{Q}_{p} \cong Q \otimes \mathbf{z}_{p} \mathbf{Q}_{p}$. Hence, $P \otimes \mathbf{z}_{p} \mathbf{F}_{p} \cong Q \otimes \mathbf{z}_{p} \mathbf{F}_{p}$.

Let $T_{\infty}$ be union of $T$ and all of infinite places $\left\{v_{0,1}, \cdots, v_{0, r}\right\}$ of $k$. For each $v_{0} \in T_{\infty}$, we choose a prolongation $v$ onto $K$ and fix it once for all. Denote by $v_{i}$ the prolongation of $v_{0, i}$. Let $\mathfrak{g}_{v} \subset \mathfrak{g}$ be the decomposition group of $v$. Denote by $\mathbf{Z} \mathfrak{g} / \mathfrak{g}_{v}$ a left $\mathbf{Z} \mathfrak{g}$-module

$$
\bigoplus_{\bar{\sigma} \in \mathfrak{g} / \mathfrak{g}_{v}} \mathbf{Z} \bar{\sigma}
$$

where $\bar{\sigma}$ denotes a coset $\sigma \mathfrak{g}_{v}$. Similarly, we denote by $\mathbf{F}_{p} \mathfrak{g} / \mathfrak{g}_{v}$ a left $R$-module generated by $\bar{\sigma}$. We see $\mathbf{Z} \mathfrak{g} / \mathfrak{g}_{v} \otimes \mathbf{F}_{p}=\mathbf{F}_{p} \mathfrak{g} / \mathfrak{g}_{v}$.

We have a system of Minkowsky's units of the relative Galois field $K / k$. Namely, there exist units $H_{i}$ of $K$ such that

$$
\begin{aligned}
\left|H_{i}\right|_{v_{i}} & >1, \quad\left|H_{i}\right|_{\sigma v_{j}}<1 \quad \text { if } i \neq j \text { or } \sigma \notin \mathfrak{g}_{v_{i}}, \\
\sigma H_{i} & =H_{i} \quad \text { for } \sigma \in \mathfrak{g}_{v_{i}},
\end{aligned}
$$

where $|\cdot|_{v_{i}}$ is the multiplicative valuation belonging to $v_{i}$. Let $F$ be a $\mathfrak{g}$ submodule of the unit group $E_{\emptyset}$ generated by $\left\{H_{i}\right\}_{i=1}^{r}$. Let $\mu_{K}$ be the torsion submodule of $E_{\emptyset}$. We have $\left(E_{\emptyset}: F \mu_{K}\right)<\infty$. Put $W_{\infty}=\oplus_{i=1}^{r} \mathbf{Z} \mathfrak{g} / \mathfrak{g}_{v_{i}}$. Denote by $\bar{H}_{i}$ the image of $H_{i}$ into $F \mu_{K} / \mu_{K}$. We have a surjective $\mathfrak{g}$ homomorphism of $W_{\infty}$ onto $F \mu_{K} / \mu_{K}$ :

$$
\ell_{\infty}:\left(\sum_{\bar{\sigma} \in \mathfrak{g} / \mathfrak{g}_{v_{i}}} m_{i, \bar{\sigma}} \bar{\sigma}: 1 \leq i \leq r\right) \quad \longrightarrow \quad \prod_{i=1}^{r} \prod_{\bar{\sigma} \in \mathfrak{g} / \mathfrak{g}_{v_{i}}} \sigma \bar{H}_{i}^{m_{i, \bar{\sigma}}}
$$

Put $e_{i}=\sum_{\bar{\sigma} \in \mathfrak{g} / \mathfrak{g}_{i}} \tilde{\sigma} \in \mathbf{Z} \mathfrak{g}$, where $\tilde{\sigma}$ denotes a representative of $\bar{\sigma} . h_{i}=e_{i} H_{i}$ is a unit of $k$ and there is a non-trivial relation

$$
1=\prod_{i=1}^{r} h_{i}^{m_{i}}
$$

Put $e=\left(m_{i} e_{i}: 1 \leq i \leq r\right) \in W_{\infty}$. We see ker $\ell_{\infty} \supset \mathbf{Z g} e \neq 0$. Since the $\mathbf{Z}$-rank of $E_{\emptyset}$ equals $\sum_{i=1}^{r}\left(\mathfrak{g}: \mathfrak{g}_{v_{i}}\right)-1$ and since the action of $\mathfrak{g}$ on $\mathbf{Z} \mathfrak{g} e$ is trivial, we have ker $\ell_{\infty}$ is a trivial $\mathfrak{g}$-module of rank 1 . Set $P=E_{\emptyset} / \mu_{K} \otimes \mathbf{Z}_{p}$ 
and $Q=\left(W_{\infty} \otimes \mathbf{Z}_{p}\right) /\left(\operatorname{ker} \ell_{\infty} \otimes \mathbf{Z}_{p}\right)$ in Lemma 8. Since $W_{\infty} /$ ker $\ell_{\infty}$ is torsion free, we have

$$
E_{\emptyset} / \mu_{K} \otimes \mathbf{F}_{p} \cong W_{\infty} \otimes \mathbf{F}_{p} / \operatorname{ker} \ell_{\infty} \otimes \mathbf{F}_{p} \cong\left(\bigoplus_{i=1}^{r} \mathbf{F}_{p} \mathfrak{g} / \mathfrak{g}_{v_{i}}\right) / \mathbf{F}_{p}
$$

Similarly, $E_{T}$ contains an element $H_{v}$ for each $v_{0} \in T$ such that $v\left(H_{v}\right)>0$ and $u\left(\sigma H_{v}\right)=0$ if $v \neq u$ or if $\sigma \notin \mathfrak{g}_{v}$. Let $F_{T}$ be a $\mathfrak{g}$-submodule of $E_{T}$ generated by $\left\{H_{v}: v_{0} \in T\right\}$ and $E_{\emptyset}$. We have $\left(E_{T}: F_{T}\right)<\infty$ and the $\mathbf{Z}$-rank of $F_{T} / E_{\emptyset}$ equals $\sum_{v_{0} \in T}\left(\mathfrak{g}: \mathfrak{g}_{v}\right)$. Put $W_{T}=\oplus_{v_{0} \in T} \mathbf{Z} \mathfrak{g} / \mathfrak{g}_{v}$. By considering a surjective homomorphism $W_{T} \rightarrow F_{T} / E_{\emptyset}$, we obtain $W_{T} \cong F_{T} / E_{\emptyset}$. Set $P=E_{T} / E_{\emptyset} \otimes \mathbf{Z}_{p}$ and $Q=W_{T} \otimes \mathbf{Z}_{p}$ in Lemma 8. We have

$$
E_{T} / E_{\emptyset} \otimes \mathbf{F}_{p} \cong \bigoplus_{v_{0} \in T} \mathbf{F}_{p} \mathfrak{g} / \mathfrak{g}_{v}
$$

Denote by $R_{v}$ the group $\operatorname{ring} \mathbf{F}_{p} \mathfrak{g}_{v}$. Let $\operatorname{ind}_{v} \mathbf{F}_{p}$ be the induced module $\operatorname{ind}_{\mathfrak{g}_{v}}^{\mathfrak{g}} \mathbf{F}_{p}=R \otimes_{R_{v}} \mathbf{F}_{p}$ of the trivial $R_{v}$-module $\mathbf{F}_{p}$. Note $\operatorname{ind}_{v} \mathbf{F}_{p}=\mathbf{F}_{p} \mathfrak{g} / \mathfrak{g}_{v}$. Let $\varepsilon_{v_{0}}$ be a character afforded with $\operatorname{ind}_{v} \mathbf{F}_{p}$. Since the sequences

$$
\begin{aligned}
& 0 \rightarrow \mu_{K} \otimes \mathbf{F}_{p} \rightarrow E_{\emptyset} \otimes \mathbf{F}_{p} \rightarrow E_{\emptyset} / \mu_{K} \otimes \mathbf{F}_{p} \rightarrow 0 \\
& 0 \rightarrow E_{\emptyset} \otimes \mathbf{F}_{p} \rightarrow E_{T} \otimes \mathbf{F}_{p} \rightarrow E_{T} / E_{\emptyset} \otimes \mathbf{F}_{p} \rightarrow 0
\end{aligned}
$$

are exact, we have

$$
\beta_{T}=\omega+\sum_{v_{0} \in T_{\infty}} \varepsilon_{v_{0}}-\varepsilon
$$

from the above isomorphisms.

Let $U_{v}^{(1)}$ be the group of principal units of $K_{v}$ for $v \in P(K)$ and $\mu_{v}$ be the $p$-primary torsion subgroup of $U_{v}^{(1)} . U_{v} \otimes \mathbf{F}_{p} \cong U_{v} / U_{v}^{p}$ is isomorphic to $U_{v}^{(1)} \otimes \mathbf{F}_{p}$ and $U_{v}^{(1)} / \mu_{v}$ is a torsion free $\mathbf{Z}_{p}$-module. Thus, the sequence

$$
0 \rightarrow \mu_{v} \otimes \mathbf{F}_{p} \rightarrow U_{v} \otimes \mathbf{F}_{p} \rightarrow U_{v}^{(1)} / \mu_{v} \otimes \mathbf{F}_{p} \rightarrow 0
$$

is exact. The $p$-adic logarithm maps $U_{v}^{(1)}$ into $K_{v}$, which is a $\mathfrak{g}_{v}$-homomorphism. Let $\mathfrak{p}_{v}$ be the valuation ideal of $K_{v}$. There is $m \geq 1$ such that the $p$-adic exponential function converges on $\mathfrak{p}_{v}^{m}$. Let $b$ be a normal basis of $K_{v} / k_{v_{0}}$ and $\left\{b_{1}, \cdots, b_{m_{v_{0}}}\right\}$ be a $\mathbf{Q}_{p}$-basis of $k_{v_{0}}$, where $m_{v_{0}}=\left[k_{v_{0}}: \mathbf{Q}_{p}\right]$. We have

$$
K_{v}=\bigoplus_{i=1}^{m_{v_{0}}} \mathbf{Q}_{p} \mathfrak{g}_{v}\left(b b_{i}\right)
$$


We may assume $b b_{i} \in \mathfrak{p}_{v}^{m}$. Let $F_{v}^{\prime}$ be a $\mathbf{Z}_{p} \mathfrak{g}_{v}$-submodule of $\mathfrak{p}_{v}^{m}$ generated by $b b_{1}, \cdots, b b_{m_{v_{0}}}$. We have $F_{v}^{\prime} \cong\left(\mathbf{Z}_{p} \mathfrak{g}_{v}\right)^{m_{v_{0}}}$. Let $F_{v}$ be the inverse image of $F_{v}^{\prime}$ in $U_{v}^{(1)}$ by the $p$-adic logarithm. We have

$$
F_{v} \mu_{v} / \mu_{v} \cong F_{v}^{\prime} \cong\left(\mathbf{Z}_{p} \mathfrak{g}_{v}\right)^{m_{v_{0}}}
$$

Since $\left(U_{v}^{(1)}: F_{v}\right)<\infty$, we obtain $U_{v}^{(1)} / \mu_{v} \otimes \mathbf{z}_{p} \mathbf{F}_{p} \cong R_{v}^{m_{v_{0}}}$ by putting $P=$ $U_{v}^{(1)} / \mu_{v}$ and $Q=F_{v} \mu_{v} / \mu_{v}$ in Lemma 8 . Note $U_{v}^{(1)} / \mu_{v} \otimes \mathbf{F}_{p} \cong U_{v}^{(1)} / \mu_{v} U_{v}^{(1) p} \cong$ $U_{v} / \mu_{v} \otimes_{\mathbf{Z}} \mathbf{F}_{p}$. Hence, we have

$$
U_{v} \otimes \mathbf{F}_{p} \cong\left(\mu_{v} \otimes \mathbf{F}_{p}\right) \oplus R_{v}^{m_{v_{0}}}
$$

Denote by $\omega_{v_{0}}$ a character afforded with $\operatorname{ind}_{v} \mu_{v} \otimes \mathbf{F}_{p} \cong \operatorname{ind}_{v} \mathbf{T}$. Let $\varphi_{\mathfrak{g}}$ denote the character of the left regular representation on $R$. Since ind $R_{v}=$ $R$ and $\prod_{u \mid v_{0}} U_{u} \otimes \mathbf{F}_{p} \cong \operatorname{ind}_{v} U_{v} \otimes \mathbf{F}_{p}$, the character of representation on $\prod_{u \mid v_{0}} U_{u} \otimes \mathbf{F}_{p}$ is $\omega_{v_{0}}+m_{v_{0}} \varphi_{\mathfrak{g}}$. Since $K_{v}^{\times} / U_{v} \cong \mathbf{Z}$, we have $\operatorname{ind}_{v}\left(K_{v}^{\times} / U_{v}\right) \otimes \mathbf{F}_{p}$ affords $\varepsilon_{v_{0}}$. Thus, the character of the representation on $\prod_{u \mid v_{0}} K_{v}^{\times} \otimes \mathbf{F}_{p}$ is $\omega_{v_{0}}+m_{v_{0}} \varphi_{\mathfrak{g}}+\varepsilon_{v_{0}}$ for $v_{0} \in P \cap T$. If $v_{0} \in S$, we denote by $\mu_{u}$ the torsion subgroup of $K_{u}^{\times}$for $u \mid v_{0}$ and have $\prod_{u \mid v_{0}} \mu_{u}^{\times} \otimes \mathbf{F}_{p}$ affords $\omega_{v_{0}}$. Note $\omega_{v_{0}}=0$ if $p \neq 2$. We obtain

$$
\kappa_{1}=[k: \mathbf{Q}] \varphi_{\mathfrak{g}}+\sum_{v_{0} \in P} \omega_{v_{0}}+\sum_{v_{0} \in P \cap T} \varepsilon_{v_{0}}+\sum_{v_{0} \in S} \omega_{v_{0}}
$$

because of $\sum_{v_{0} \in P} m_{v_{0}}=[k: \mathbf{Q}]$.

We have an isomorphism of $R$-modules:

$$
V / U^{p} \cong \bigoplus_{v_{0} \in P \backslash T} \operatorname{ind}_{v} V_{v} / U_{v}^{p} \oplus \bigoplus_{v_{0} \in P \cap T} \operatorname{ind}_{v} V_{v} / K_{v}^{\times p} .
$$

Let $W_{1}$ (resp. $W_{2}$ ) be the submodule generated by $V_{v}$ (resp. $V_{v} \cap U_{v}$ ) in $K_{v}^{\times} \otimes \mathbf{F}_{p}$ (resp. $U_{v} \otimes \mathbf{F}_{p}$ ). By inclusion $U_{v} \otimes \mathbf{F}_{p} \subset K_{v}^{\times} \otimes \mathbf{F}_{p}$, we consider $W_{2}$ a submodule of $W_{1}$. Since $K_{v}^{\times} / U_{v} \cong \mathbf{Z}$, we have $W_{1} / W_{2} \cong \mathbf{F}_{p}$ if $v_{0} \in P \cap T$. Note $W_{1}=W_{2}$ if $v_{0} \in P \backslash T$. Let $W_{3}$ be a submodule of $U_{v} \otimes \mathbf{F}_{p}$ generated by $\xi_{v} \otimes 1$ over $R_{v}$. Since $K_{v}\left(\sqrt[p]{\xi_{v}}\right)$ is compositum of $K_{v}$ and the unramified abelian extension of degree $p$ over $k_{v_{0}}$, we see $\operatorname{Gal}\left(K_{v}\left(\sqrt[p]{\xi_{v}}\right) / K_{v}\right) \cong \mathbf{F}_{p}$ as $R_{v}$-modules. By the Kummer duality, this means $W_{3} \cong \mathbf{T}$. Put $W_{v}=K_{v}^{\times}$ if $v_{0} \in P \cap T$, and $W_{v}=U_{v}$ for $v_{0} \in P \backslash T$. We have $\operatorname{dim} W_{3}=1$ and $\left(V_{v} / W_{v}^{p}\right) \cong\left(W_{1} / W_{2}\right) \oplus\left(W_{2} / W_{3}\right) \oplus W_{3}$. By (3.4), we have

$$
R_{v}^{m_{v_{0}}} \cong\left(U_{v} \otimes \mathbf{F}_{p}\right) / W_{3} \supset W_{2} / W_{3} .
$$


Let $\delta_{v_{0}}$ be the character afforded with $\operatorname{ind}_{v_{0}} W_{2} / W_{3}$. We have

$$
\kappa_{2}=\sum_{v_{0} \in P}\left(\omega_{v_{0}}+\delta_{v_{0}}\right)+\sum_{v_{0} \in P \cap T} \varepsilon_{v_{0}}, \quad \delta_{v_{0}} \leq m_{v_{0}} \varphi_{\mathfrak{g}}
$$

We have the following formula from (3.5), (3.6) and (2.6):

$$
\begin{aligned}
& \kappa_{1}-\kappa_{2}=[k: \mathbf{Q}] \varphi_{\mathfrak{g}}-\sum_{v_{0} \in P} \delta_{v_{0}}+\sum_{v_{0} \in S} \omega_{v_{0}}, \\
& \kappa_{1}-\kappa_{2}-\beta_{T}+\varphi_{B_{1}} \geq \alpha
\end{aligned}
$$

where $\varphi_{B_{1}}$ is the character of the representation onto $B_{1}$.

\section{$\S 4$. The value of $a_{\Phi^{*}}$}

We denote by $\varphi(\Phi)$ the coefficient of $\Phi$ for an element $\varphi$ of $\operatorname{ch}(R)$. If $\varphi$ is a character afforded with an $R$-module $Y$, we have

$$
\varphi(\Phi)=r_{\Phi}(Y)=\frac{1}{f_{\Phi}} \operatorname{dim} \operatorname{Hom}_{R}\left(L_{\Phi}, Y\right)=\frac{1}{f_{\Phi}} \operatorname{dim} \operatorname{Hom}_{R}\left(Y, L_{\Phi}\right)
$$

because of $f_{\Phi}=\operatorname{dim} \operatorname{Hom}_{R}\left(L_{\Phi}, L_{\Phi}\right)$. We recall characters:

$$
\begin{array}{cl}
\text { characters } & \text { modules } \\
\alpha & \text { coker }\left(C_{T} \stackrel{\rho}{\rightarrow} \operatorname{Gal}\left(K_{1} / K_{0}\right)=U / \iota\left(E_{T}\right) V\right) \\
\beta_{T} & E_{T} \otimes \mathbf{F}_{p} \\
\gamma_{T} & C_{T} \\
\delta_{v_{0}} & \operatorname{ind}_{v}\left(V_{v} \cap U_{v} / U_{v}^{p}<\xi_{v}>\right) \text { for } v_{0} \in P \\
\varepsilon_{v_{0}} & \operatorname{ind}_{v} \mathbf{F}_{p} \text { for } v \mid v_{0}
\end{array}
$$

Let $\theta$ be the character afforded with $\Theta$.

THEOREM 9. The value of $a_{\Phi^{*}}=r_{\Phi^{*}}\left(\mathcal{C} \otimes \mathbf{F}_{p} / J \mathcal{C} \otimes \mathbf{F}_{p}\right)$ satisfies an inequality

$$
\begin{aligned}
& \beta_{T}(\Phi)+\gamma_{T}(\Phi)+\theta\left(\Phi^{*}\right) \\
\geq & a_{\Phi^{*}} \\
\geq & \alpha(\Phi)+\beta_{T}(\Phi)+\gamma_{T}(\Phi)+\theta\left(\Phi^{*}\right)+\sum_{v_{0} \in P} \delta_{v_{0}}(\Phi) \\
& \quad-\sum_{v_{0} \in S} \omega_{v_{0}}(\Phi)-[k: \mathbf{Q}] d_{\Phi}-r_{\Phi^{*}}\left(\operatorname{Gal}\left(M^{\mathrm{ab}} / K\right)\right) .
\end{aligned}
$$

Moreover, we have the following statements: 
(1) If $\tilde{1}_{\Phi^{*}} \operatorname{Gal}\left(M^{\mathrm{ab}} / K\right)=0$ or if $\tilde{1}_{\Phi^{*}} \operatorname{Gal}\left(H^{\mathrm{ab}} / K\right)^{p}=0$, the value of $a_{\Phi^{*}}$ equals

$$
\begin{aligned}
\alpha(\Phi)+ & \sum_{v_{0} \in T_{\infty}} \varepsilon_{v_{0}}(\Phi)+\omega(\Phi)-\varepsilon(\Phi)+\gamma_{T}(\Phi)+\theta\left(\Phi^{*}\right)+\sum_{v_{0} \in P} \delta_{v_{0}}(\Phi) \\
& -\sum_{v_{0} \in S} \omega_{v_{0}}(\Phi)-[k: \mathbf{Q}] d_{\Phi}-r_{\Phi^{*}}\left(\operatorname{Gal}\left(M^{\mathrm{ab}} / K\right)\right)
\end{aligned}
$$

(2) If $G=\mathfrak{g} \times G_{p}$, we have $\tilde{1}_{\Phi^{*}} \operatorname{Gal}\left(M^{\mathrm{ab}} / K\right)$ and $\tilde{1}_{\Phi^{*}} H^{-3}\left(G_{p}, \mathbf{Z}_{p}\right)$ vanish for $\Phi^{*} \neq \varepsilon$.

(3) Suppose $p>2$. If $\delta_{v_{0}}(\Phi)=m_{v_{0}} d_{\Phi}$ for every $v_{0} \in P$, we have $\alpha(\Phi)=0$.

Proof. The inequality and the statement (1) follow from Theorem 3, 7 and formulas $(3.7),(3.3)$. If $G=\mathfrak{g} \times G_{p}$, we have $\mathfrak{g}$ acts trivially on $G_{p}$ with conjugation. Hence, $\operatorname{Gal}\left(M^{\mathrm{ab}} / K\right)$ and $H^{-3}\left(G_{p}, \mathbf{Z}_{p}\right)$ are trivial $\mathfrak{g}$-modules. Hence,

$$
\tilde{1}_{\Phi^{*}} \operatorname{Gal}\left(M^{\mathrm{ab}} / K\right)=0, \quad \tilde{1}_{\Phi^{*}} H^{-3}\left(G_{p}, \mathbf{Z}_{p}\right)=0
$$

whenever $\Phi^{*} \neq \varepsilon$. This proves (2). Suppose $p$ is odd. We see $\omega_{v_{0}}=0$ for $v_{0} \in$ $S$. If $\delta_{v_{0}}(\Phi)=m_{v_{0}} d_{\Phi}$ for every $v_{0} \in P$, we have $\sum_{v_{0} \in P} \delta_{v_{0}}(\Phi)=[k: \mathbf{Q}] d_{\Phi}$. By (3.7), we have $\alpha(\Phi) \leq \kappa_{1}(\Phi)-\kappa_{2}(\Phi)=0$, because of $\beta_{T}-\varphi_{B_{1}} \geq 0$.

We denote by $U_{M}, V_{M}$ the closed subgroup of the idele group of $K$ defined by (2.3) and (2.5) with adding subscript to specify the field $M$. We also denote by $\rho_{M}$ the map defined in (2.7). We write $B_{M}, B_{1, M}, B_{0, M}$ for $B, B_{1}, B_{0}$.

LEMMA 10. Let $p$ be an odd prime. If $k_{v_{0}} \not \supset \zeta_{p}$ and $\mathfrak{g}_{v}$ acts on $\operatorname{Gal}\left(M_{w}^{\mathrm{ab}} / K_{v}\right)$ trivially for every $v_{0} \in P$, we have $T \cap P=\emptyset$. Moreover, we have an $R_{v}$-isomorphism $V_{v} / U_{v}^{p} \cong \mathbf{T}^{c}$ for $c=\operatorname{dim} \operatorname{Gal}\left(M_{w}^{\mathrm{ab}}\left(\sqrt[p]{\xi_{v}^{\prime}}\right) \cap\right.$ $\left.K_{v}^{*} / K_{v}\right)$.

Proof. By (2.3), we see $V_{v} K_{v}^{\times p} / K_{v}^{\times p}$ is the Kummer radical of a Kummer extension $M_{w}^{\mathrm{ab}}\left(\sqrt[p]{\xi_{v}^{\prime}}\right) \cap K_{v}^{*} / K_{v}$. Let $\mathbf{Q}_{p}^{u r}$ be the maximal unramified abelian $p$-extension of $\mathbf{Q}_{p}$. Since $M_{w}^{\mathrm{ab}}\left(\sqrt[p]{\xi_{v}^{\prime}}\right)$ is a subfield $\mathbf{Q}_{p}^{u r} M_{w}^{\mathrm{ab}}$ and since $\mathfrak{g}_{v}$ acts trivially on $\operatorname{Gal}\left(\mathbf{Q}_{p}^{u r} M_{w}^{\mathrm{ab}} / K_{v}\right)$ with conjugation, we have $\operatorname{Gal}\left(M_{w}^{\mathrm{ab}}\left(\sqrt[p]{\xi_{v}^{\prime}}\right) / K_{v}\right)$ is a trivial $\mathfrak{g}_{v}$-module. Thus, considering the Kummer pairing, the Kummer radical is isomorphic to $\mathbf{T}^{c}$ for $c=$ $\operatorname{dim} \operatorname{Gal}\left(M_{w}^{\mathrm{ab}}\left(\sqrt[p]{\xi_{v}^{\prime}}\right) \cap K_{v}^{*} / K_{v}\right)$. Note $\mathbf{T} \approx \mathbf{F}_{p}$ as $\mathfrak{g}_{v}$-modules, because of $k_{v_{0}} \not \supset$ 
$\zeta_{p}$. Since $K_{v}^{\times} / U_{v} K_{v}^{\times p}$ is a trivial $\mathfrak{g}_{v}$-module, the image of $V_{v} K_{v}^{\times p} / K_{v}^{\times p} \rightarrow$ $K_{v}^{\times} / U_{v} K_{v}^{\times p}$ is 0 in an exact sequence

$$
0 \rightarrow V_{v} \cap U_{v} / U_{v}^{p} \rightarrow V_{v} K_{v}^{\times p} / K_{v}^{\times p} \rightarrow K_{v}^{\times} / U_{v} K_{v}^{\times p}
$$

Hence, $V_{v} \subset U_{v} K_{v}^{\times p}$. This proves $T \cap P=\emptyset$.

Lemma 11. Let $p$ be an odd prime. Suppose that $M$ is an abelian extension of $\mathbf{Q}$ containing $\zeta_{p^{t}}$ for $t \geq 2$ such that $K_{v}\left(\zeta_{p^{t}}\right)$ is ramified over $K_{v}$ for every $v_{0} \in P$. Furthermore, we also suppose $k_{v_{0}} \not \supset \zeta_{p}$ for every $v_{0} \in P$. Put $N=M \cap \cup_{n \geq 1} K\left(\zeta_{p^{n}}\right)$. We have a commutative diagram

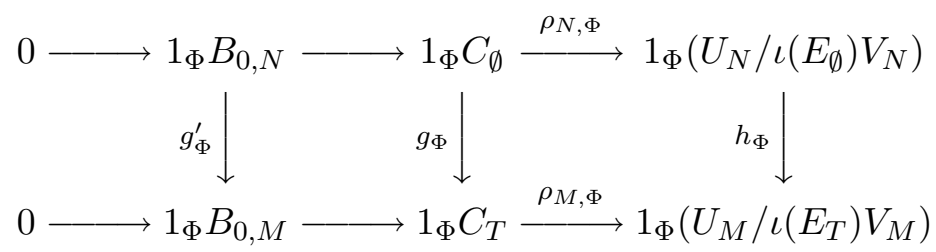

where $\rho_{M, \Phi}$ and $\rho_{N, \Phi}$ denote restriction onto the $1_{\Phi}$-components, respectively. Moreover, we have

$$
\sum_{v_{0} \in T} \varepsilon_{v_{0}}(\Phi) \geq r_{\Phi}\left(\operatorname{ker} h_{\Phi}\right)=\sum_{v_{0} \in T} \varepsilon_{v_{0}}(\Phi)-r_{\Phi}\left(B_{1, M}\right)+r_{\Phi}\left(B_{1, N}\right) .
$$

If $\sum_{v_{0} \in T} \varepsilon_{v_{0}}(\Phi)=0$, we have $r_{\Phi}\left(B_{M}\right)=r_{\Phi}\left(B_{N}\right)$.

Proof. By Lemma 10, we have $T \cap P=\emptyset$. Hence, $U_{M}=U_{N}$. To prove the lemma, we need to show $V_{M}=V_{N}$. Denote by $V_{M, v}$ be the $v$ component of the direct product $V_{M}=\prod_{v \in P(K)} V_{v}$. We see $V_{M, v} \subset U_{v}$. Since $\mathbf{Q}_{p}^{u r} M_{w}$ contains $M_{w}\left(\sqrt[p]{\xi_{v}^{\prime}}\right)$ and is abelian over $\mathbf{Q}_{p}$, there are $m \geq t$, $a>0$ and $b>0$ such that $a$ is prime to $p$ and so that $\mathbf{Q}_{p}\left(\zeta_{p^{m}}, \zeta_{p^{a p^{b}}-1}\right)$ contains $M_{w}\left(\sqrt[p]{\xi_{v}^{\prime}}\right)$. The $p$-Sylow subgroup of $\operatorname{Gal}\left(\mathbf{Q}_{p}\left(\zeta_{p^{m}}, \zeta_{p^{a p^{b}}-1}\right) / \mathbf{Q}_{p}\right)$ is isomorphic to $\mathbf{Z} / p^{m-1} \mathbf{Z} \times \mathbf{Z} / p^{b} \mathbf{Z}$. Hence, $\operatorname{Gal}\left(M_{w}\left(\sqrt[p]{\xi_{v}^{\prime}}\right) / K_{v}\right)$ is an abelian $p$-group generated by one or two elements. Let $p^{e}$ be the ramification index of $K_{v}\left(\zeta_{p^{t}}\right) / K_{v}$. We see

$$
\operatorname{Gal}\left(K_{v}^{\prime}\left(\zeta_{p^{t}}, \sqrt[p]{\xi_{v}^{\prime}}\right) / K_{v}^{\prime}\right) \cong \mathbf{Z} / p \mathbf{Z} \times \mathbf{Z} / p^{e} \mathbf{Z}
$$

because of $K_{v}^{\prime}\left(\zeta_{p^{t}}\right) \cap K_{v}^{\prime}\left(\sqrt[p]{\xi_{v}^{\prime}}\right)=K_{v}^{\prime}$, where $K_{v}^{\prime}$ is the inertia field of $M_{w} / K_{v}$. This means $\operatorname{Gal}\left(M_{w}\left(\sqrt[p]{\xi_{v}^{\prime}}\right) / K_{v}\right)$ is not cyclic. We have an $R_{v}$-isomorphism

$$
\operatorname{Gal}\left(M_{w}\left(\sqrt[p]{\xi_{v}^{\prime}}\right) \cap K_{v}^{*} / K_{v}\right) \cong \mathbf{F}_{p}^{2}
$$


Therefore, by Lemma 10, we have

$$
V_{M, v} / U_{v}^{p} \cong \mathbf{T}^{2}
$$

In particular, we have $V_{N, v} / U_{v}^{p} \cong \mathbf{T}^{2}$ if $M=N$. Suppose $M \neq N$. Let $u$ be restriction of $w$ onto $N$ and $K_{v}^{\prime \prime}$ be the inertia field of $N_{u} / K_{v}$. Let $K_{v}^{\prime \prime}\left(\sqrt[p]{\xi_{v}^{\prime \prime}}\right)$ be an unramified cyclic extension of degree $p$ over $K_{v}^{\prime \prime}$. Since $M_{w}\left(\sqrt[p]{\xi_{v}^{\prime}}\right) \supset N_{u}\left(\sqrt[p]{\xi_{v}^{\prime \prime}}\right)$, we have $V_{M, v} \supset V_{N, v} \cdot V_{M, v}=V_{N, v}$ follows from isomorphisms $V_{M, v} / U_{v}^{p} \cong V_{N, v} / U_{v}^{p} \cong \mathbf{T}^{2}$. This proves $V_{M}=V_{N}$.

Denote by $U$ (resp. $V$ ) the group $U_{M}=U_{N}\left(\operatorname{resp} . V_{M}=V_{N}\right)$ with omitting the subscript. Let $Y$ be a submodule of $D_{\emptyset}$ generated by $\left\{\sigma \mathfrak{p}_{v}: \sigma \in\right.$ $\left.\mathfrak{g}, v_{0} \in T\right\}$. We have $D_{\emptyset}=D_{T} \oplus Y$. Denote by $\mathfrak{a}=\mathfrak{a}^{\prime}+\mathfrak{a}^{\prime \prime}$ the decomposition of $\mathfrak{a} \in D_{\emptyset}$ into a sum of $\mathfrak{a}^{\prime} \in D_{\emptyset}$ and $\mathfrak{a}^{\prime \prime} \in Y$. A homomorphism $D_{\emptyset} / P_{\emptyset} \rightarrow$ $D_{T} / P_{T}$ is induced from $\mathfrak{a} \rightarrow \mathfrak{a}^{\prime}$. Let $g: C_{\emptyset} \rightarrow C_{T}$ be restriction of this homomorphism onto the $p$-torsion submodules. Denote by $h$ a canonical map $U / \iota\left(E_{\emptyset}\right) V \rightarrow U / \iota\left(E_{T}\right) V$. Let $g_{\Phi}$ (resp. $\left.h_{\Phi}\right)$ be restriction of $g$ (resp. $h$ ) onto the $\Phi$-component. Put $g_{\Phi}^{\prime}=g_{\Phi \mid 1_{\Phi} B_{0, N}}$. Since $\mathfrak{a}^{p}=(a)$ in $D_{\emptyset}$ for $a \in K^{\times}$implies $\mathfrak{a}^{\prime p}=(a)$ in $D_{T}$ and since every element $c \in C_{\emptyset}$ contains $\mathfrak{a} \in D_{\emptyset}$ such that $(\mathfrak{a}, p)=1$, the commutativity of the diagram follows from the definition (2.7) of the maps $\rho_{M}$ and $\rho_{N}$.

We have $B_{1, N} \cong E_{\emptyset} \cap \iota^{-1}(V) / E_{\emptyset}^{p}$ and $B_{1, M} \cong E_{T} \cap \iota^{-1}(V) / E_{T}^{p}$. Observe

$$
\begin{aligned}
\operatorname{ker} h=\iota\left(E_{T}\right) V / \iota\left(E_{\emptyset}\right) V & \cong \iota\left(E_{T}\right) /\left(\iota\left(E_{T}\right) \cap V\right) \iota\left(E_{\emptyset}\right) \\
& \cong E_{T} /\left(E_{T} \cap \iota^{-1}(V)\right) E_{\emptyset},
\end{aligned}
$$

because of $\operatorname{ker} \iota \cap E_{T} \subset E_{T} \cap \iota^{-1}(V)$. Therefore, we obtain exact sequences

$$
\begin{aligned}
& 0 \rightarrow B_{1, N} \rightarrow B_{1, M} \rightarrow\left(E_{T} \cap \iota^{-1}(V)\right) /\left(E_{\emptyset} \cap \iota^{-1}(V)\right) E_{T}^{p} \rightarrow 0, \\
& 0 \rightarrow\left(E_{T} \cap \iota^{-1}(V)\right) /\left(E_{\emptyset} \cap \iota^{-1}(V)\right) E_{T}^{p} \rightarrow E_{T} / E_{\emptyset} E_{T}^{p} \rightarrow \operatorname{ker} h \rightarrow 0 .
\end{aligned}
$$

Thus, $r_{\Phi}(\operatorname{ker} h) \leq \sum_{v_{0} \in T} \varepsilon_{v_{0}}(\Phi)$ follows from (3.2). Furthermore, we also have the formula of $r_{\Phi}($ ker $h)$ from these sequences.

Suppose $\sum_{v_{0} \in T} \varepsilon_{v_{0}}(\Phi)=0$. By virtue of the formula, we obtain ker $h_{\Phi}=$ 0 and $r_{\Phi}\left(B_{1, N}\right)=r_{\Phi}\left(B_{1, M}\right)$. Thus, $h_{\Phi}$ is an isomorphism. Since $D_{T} / P_{T} \cong$ $D_{\emptyset} / P_{\emptyset} Y$, we have an exact sequence

$$
\tilde{1}_{\Phi} Y \otimes \mathbf{Z}_{p} \rightarrow \tilde{1}_{\Phi} D_{\emptyset} / P_{\emptyset} \otimes \mathbf{Z}_{p} \rightarrow \tilde{1}_{\Phi} D_{T} / P_{T} \otimes \mathbf{Z}_{p} \rightarrow 0 .
$$

Denote by $\tilde{\mathfrak{p}}_{v}$ an element $\mathfrak{p}_{v} \otimes 1$ of $Y \otimes \mathbf{Z}_{p}$. We see $Y \otimes \mathbf{Z}_{p}=\oplus_{v_{0} \in T} A \cdot \tilde{\mathfrak{p}}_{v}$. Since $A \cdot \tilde{\mathfrak{p}}_{v} \cong A \otimes \mathbf{z}_{p} \mathfrak{g}_{v} \mathbf{Z}_{p}$ and $1_{\Phi}\left(R \otimes R_{v} \mathbf{F}_{p}\right) \cong L_{\Phi}^{\varepsilon_{v_{0}}}(\Phi)$, we have $1_{\Phi}\left(A \cdot \tilde{\mathfrak{p}}_{v} \otimes \mathbf{F}_{p}\right)=0$. 
This implies $\tilde{1}_{\Phi}\left(A \cdot \tilde{\mathfrak{p}}_{v}\right)=0$. Hence, $\tilde{1}_{\Phi} Y \otimes \mathbf{Z}_{p}=0$. We see $\tilde{1}_{\Phi} D_{\emptyset} / P_{\emptyset} \otimes \mathbf{Z}_{p} \cong$ $\tilde{1}_{\Phi} D_{T} / P_{T} \otimes \mathbf{Z}_{p}$. Since $1_{\Phi} C_{\emptyset}$ (resp. $1_{\Phi} C_{T}$ ) is the $p$-torsion submodule of $\tilde{1}_{\Phi} D_{\emptyset} / P_{\emptyset} \otimes \mathbf{Z}_{p}$ (resp. $\tilde{1}_{\Phi} D_{T} / P_{T} \otimes \mathbf{Z}_{p}$ ), we have $1_{\Phi} C_{\emptyset} \cong 1_{\Phi} C_{T}$. Therefore, $g_{\Phi}$ is an isomorphism. By the commutative diagram, $g_{\Phi}^{\prime}$ is also an isomorphism. We obtain $r_{\Phi}\left(B_{0, N}\right)=r_{\Phi}\left(B_{0, M}\right)$. Consequently, $r_{\Phi}\left(B_{M}\right)=r_{\Phi}\left(B_{N}\right)$ holds by virtue of the formula $(2.4)$.

Proposition 12. Let notations and assumptions be same as those in Lemma 11. In addition, we suppose $k=\mathbf{Q}$ and that $\Phi$ satisfies $\tau 1_{\Phi}=1_{\Phi}$ for the complex conjugation $\tau$. Then, we have $a_{\Phi^{*}} \leq 1$ if and only if $\Phi$ satisfies one of the following conditions:

(1) $\gamma_{T}(\Phi)=\sum_{v_{0} \in T} \varepsilon_{v_{0}}(\Phi)=0$ if $\omega_{p}(\Phi)=1$,

(2) $r_{\Phi}\left(B_{1, M}\right) \leq 1$ and $\gamma_{T}(\Phi)=0$ if $\omega_{p}(\Phi)=0, \Phi \neq \varepsilon$ and $\sum_{v_{0} \in T} \varepsilon_{v_{0}}(\Phi)=$ 1 ,

(3) $\gamma_{P}\left(\Phi^{*}\right) \leq 1$ if $\omega_{p}(\Phi)=0, \Phi \neq \varepsilon$ and $\sum_{v_{0} \in T} \varepsilon_{v_{0}}(\Phi)=0$,

(4) $\sharp T+\alpha(\varepsilon) \leq 2$ if $\Phi=\varepsilon$.

Proof. We may take $t=2$ in Lemma 11. Suppose $\tau 1_{\Phi}=1_{\Phi}$. Note $\tau 1_{\Phi}=1_{\Phi}$ is equivalent to $\tau 1_{\Phi^{*}} \neq 1_{\Phi^{*}} . \tau 1_{\Phi^{*}} \neq 1_{\Phi^{*}}$ means $\Phi^{*} \neq \varepsilon$. Since $M / \mathbf{Q}$ is abelian, we have $\theta\left(\Phi^{*}\right)=r_{\Phi^{*}}\left(\operatorname{Gal}\left(M^{\mathrm{ab}} / \mathbf{Q}\right)\right)=0$ from $(2)$ of Theorem 9 . $\mathfrak{g}_{v}$ is a normal subgroup generated by $\tau$ for $v_{0}=\infty$. $\varepsilon_{\infty}$ is afforded with $\mathbf{F}_{p} \mathfrak{g} /<\tau>$. Hence, $\varepsilon_{\infty}(\Phi)=1$. By (3.3), we have

$$
\beta_{T}(\Phi)=1+\sum_{v_{0} \in T} \varepsilon_{v_{0}}(\Phi)-\varepsilon(\Phi)
$$

Let $v_{0}=p$. By (3.6) and (4.2), we have $\kappa_{2}=\delta_{p}+\omega_{p}=2 \omega_{p}$. Hence, $\delta_{p}=\omega_{p}$.

Suppose $\omega_{p}(\Phi) \neq 0$. We have $\delta_{p}(\Phi)=1$. Hence, $\Phi \neq \varepsilon$. Note $1=m_{p}=$ $d_{\Phi}$. By (1) and (3) of Theorem 9, we observe $a_{\Phi^{*}}$ equals $1+\sum_{v_{0} \in T} \varepsilon_{v_{0}}(\Phi)+$ $\gamma_{T}(\Phi)$. Thus, $a_{\Phi^{*}} \leq 1$ if and only if $\sum_{v_{0} \in T} \varepsilon_{v_{0}}(\Phi)=\gamma_{T}(\Phi)=0$.

Suppose $\omega_{p}(\Phi)=0$ and $\Phi \neq \varepsilon$. We have

$$
a_{\Phi^{*}}=\alpha(\Phi)+\sum_{v_{0} \in T} \varepsilon_{v_{0}}(\Phi)+\gamma_{T}(\Phi) .
$$

We observe that $\sum_{v_{0} \in T} \varepsilon_{v_{0}}(\Phi) \leq 1$ if $a_{\Phi^{*}} \leq 1$. Firstly, let $\Phi$ satisfy $\sum_{v_{0} \in T} \varepsilon_{v_{0}}(\Phi)=1$. If $a_{\Phi^{*}} \leq 1$, we have $\alpha(\Phi)=\gamma_{T}(\Phi)=0$. Since $\gamma_{T}(\Phi)=0$ 
implies $r_{\Phi}\left(B_{0, M}\right)=0$, we have $r_{\Phi}\left(B_{1, M}\right) \leq 1$ from (2.4). Conversely, if $r_{\Phi}\left(B_{1, M}\right) \leq 1$ and $\gamma_{T}(\Phi)=0$, we also have $a_{\Phi^{*}} \leq 1$ from (2.4). Secondly, let $\Phi$ satisfy $\sum_{v_{0} \in T} \varepsilon_{v_{0}}(\Phi)=0$. By virtue of Lemma 11, we are able to reduce to $N$. We may suppose $M=N$. Since $T \subset P$, we see $T=\emptyset$ from Lemma 10. Since $\kappa_{2}=2 \omega_{p}$, we have $1_{\Phi}\left(V / U^{p}\right)=0$. Put $W=\prod_{v \in P(K)} K_{v}^{\times}$. We also have $1_{\Phi}\left(V W^{p} / W^{p}\right)=0$. Hence, $1_{\Phi}\left(W / V W^{p}\right) \cong 1_{\Phi}\left(W / W^{p}\right)$. Observe that $a \otimes 1 \in K^{\times} \otimes \mathbf{F}_{p}$ for $(a) \in D_{\emptyset}^{p}$ is an element of $B_{M}$ if and only if the image of $a$ into $W / V W^{p}$ is equal to 0 . When $a \otimes 1 \in 1_{\Phi} B_{M}$, we have $\iota(a) \in$ $V W^{p}$ is equivalent to $\iota(a) \in W^{p}$, because of $1_{\Phi}\left(W / V W^{p}\right) \cong 1_{\Phi}\left(W / W^{p}\right)$. Namely, $K(\sqrt[p]{a}) / K$ is unramified $p$-decomposed, where we call $K(\sqrt[p]{a}) / K p$ decomposed if every place lying above $p$ is completely decomposed there. Let $K_{2}$ be the maximal unramified $p$-decomposed elementary $(p, \cdots, p)$-abelian extension of $K$. Let $B^{\prime}$ be the Kummer radical of $K_{2} / K$. By the Kummer duality, $B^{\prime}$ is dual to $D_{P} / P_{P} \otimes \mathbf{F}_{p}$. Hence, $1_{\Phi} B^{\prime}$ and $1_{\Phi^{*}} D_{P} / P_{P} \otimes \mathbf{F}_{p}$ are dual to each other. We have $\gamma_{P}\left(\Phi^{*}\right)=r_{\Phi}\left(B^{\prime}\right) .1_{\Phi} B^{\prime} \supset 1_{\Phi} B_{M}$ follows from the above argument. Since $K_{2}$ is a subfield of the maximal unramified abelian $p$-extension $H$ of $M$, we have $B_{M} \supset B^{\prime}$. We have $1_{\Phi} B_{M}=1_{\Phi} B^{\prime}$. Therefore, we obtain $a_{\Phi^{*}}=\gamma_{P}\left(\Phi^{*}\right)$.

Suppose $\Phi=\varepsilon$. We see $\Phi^{*}=\omega$. Let $M_{0}$ be the maximal $p$-extension of $\mathbf{Q}$ contained in $M$. We have $M=M_{0} K$. By Lemma 4, we are able to compute the value $a_{\omega}$ in $M_{0}\left(\zeta_{p}\right)$. Hence, suppose $M=M_{0}\left(\zeta_{p}\right)$ and $K=$ $\mathbf{Q}\left(\zeta_{p}\right)$. We have

$$
a_{\omega}=\alpha(\varepsilon)+\sum_{v_{0} \in T} \varepsilon_{v_{0}}(\varepsilon)-1+\gamma_{T}(\varepsilon) .
$$

Since the class number of $\mathbf{Q}$ is one, we have $\gamma_{T}(\varepsilon)=0$. Therefore, $a_{\omega}=\alpha(\varepsilon)+\sharp T-1$, because of $\varepsilon_{v_{0}}(\varepsilon)=\operatorname{dim}_{\operatorname{Hom}}\left(R \otimes_{R_{v}} \mathbf{F}_{p}, \mathbf{F}_{p}\right)=$ $\operatorname{dim} \operatorname{Hom}_{R_{v}}\left(\mathbf{F}_{p}, \mathbf{F}_{p}\right)=1$.

Proposition 13. Let $p$ be odd and $k$ be a totally real field. Let $M$ be a CM-field containing $\zeta_{p^{n}}$ for $n \geq 2$. Suppose $M=K\left(\zeta_{p^{n}}\right)$ and $M \neq K$. Let $h_{M}^{+}$be the class number of the maximal totally real subfield of $M$ and $h_{K}^{-}$be the relative class number of $K$. Let $\tau$ be the complex conjugation of $M$.

(1) We have $p X h_{M}^{+}$if $p \backslash h_{K}^{-}$and if $a_{\varepsilon}=0$.

(2) Suppose $k=\mathbf{Q}$ and $M / \mathbf{Q}$ is abelian. We have $p \not h_{M}^{+}$, if $\gamma_{\emptyset}\left(\Phi^{*}\right)=1$ and $\alpha\left(\Phi^{*}\right)=\omega_{p}\left(\Phi^{*}\right)=0$ for every $\Phi^{*}$ such that $\Phi^{*} \neq \omega, \tau 1_{\Phi^{*}} \neq 1_{\Phi^{*}}$ and $\gamma_{\emptyset}\left(\Phi^{*}\right)>0$. 
Proof. Let $M_{0}$ be the maximal p-extension over $\mathbf{Q}$ contained in $\mathbf{Q}\left(\zeta_{p^{n}}\right)$. Since $M=M_{0} K$, we see $G=G_{p} \times \mathfrak{g}$. Hence, $\mathcal{C}$ is a $\mathbf{Z}_{p} \mathfrak{g}$-module. Since $\mathcal{C}=\oplus_{\Phi} \tilde{1}_{\Phi} \mathcal{C}$, we have $\mathcal{C}^{\tau+1}=0$ if and only if $\tilde{1}_{\Phi} \mathcal{C}=0$ for every $\Phi$ such that $\tau 1_{\Phi}=1_{\Phi}$. Further, $\tilde{1}_{\Phi} \mathcal{C}=0$ is equivalent to $a_{\Phi}=0$. Thus, $p \nmid \backslash h_{M}^{+}$if and only if $a_{\Phi}=0$ for every $\Phi$ such that $\tau 1_{\Phi}=1_{\Phi}$.

By (2) of Theorem 9, we have $\theta(\Phi)=0$ and $\tilde{1}_{\Phi} \operatorname{Gal}\left(M^{\mathrm{ab}} / K\right)=0$ for $\Phi \neq \varepsilon$. Note $P \supset T$. We can show $T=\emptyset$. In fact, for $v \in P(K)$, if $M_{w} / K_{v}$ is unramified, we see $M_{w}\left(\sqrt[p]{\xi_{v}^{\prime}}\right) \cap K_{v}^{*}=K_{v}\left(\sqrt[p]{\xi_{v}}\right)$, because $M_{w}\left(\sqrt[p]{\xi_{v}^{\prime}}\right) / K_{v}$ is unramified. Contrarily, if $M_{w} / K_{v}$ is ramified, we have $M_{w}\left(\sqrt[p]{\xi_{v}^{\prime}}\right) / K_{v}$ is not cyclic, because $M_{w}\left(\sqrt[p]{\xi_{v}^{\prime}}\right) / M_{w}$ is an unramified extension of degree $p$. Hence $\operatorname{Gal}\left(M_{w}\left(\sqrt[p]{\xi_{v}^{\prime}}\right) / K_{v}\right) \cong \mathbf{Z} / p^{a} \mathbf{Z} \times \mathbf{Z} / p \mathbf{Z}$, where $p^{a}=\left[M_{w}: K_{v}\right]$. Let $K_{v}(\sqrt[p]{x}) / K_{v}$ be an extension of degree $p$ for $x \in K_{v}^{\times}$such that $M_{w} K_{v}(\sqrt[p]{x})=$ $M_{w}\left(\sqrt[p]{\xi_{v}^{\prime}}\right)$. Since $K_{v}^{\prime} \subset M_{w}=K_{v}\left(\zeta_{p^{n}}\right)$, there is $1 \leq e<n$ such that $K_{v}^{\prime}=K_{v}\left(\zeta_{p^{e}}\right)$. Considering the structure of the Galois group, we have $K_{v}\left(\zeta_{p^{e+1}}, \sqrt[p]{x}\right)$ contains $K_{v}^{\prime}\left(\sqrt[p]{\xi_{v}^{\prime}}\right)$. Since $\left\{\zeta_{p^{e+1}}^{p}, \sqrt[p]{x^{p}}, \sqrt[p]{\xi_{v}^{\prime}} p\right\} \subset K_{v}^{\prime}$, there are integers $0 \leq t, s<p$ such that $\xi_{v}^{\prime}=\zeta_{p^{e}}^{t} x^{s} y^{p}$ for $y \in K_{v}^{\prime \times}$. We see $x^{s}=\xi_{v}^{\prime} \zeta_{p^{e}}^{-t} y^{-p} \in K_{v}$. Note $s \neq 0$, because $K_{v}^{\prime}\left(\zeta_{p^{e+1}}\right) / K_{v}^{\prime}$ is ramified. Since $K_{v}^{\prime} / K_{v}$ is unramified, this shows that $x \in U_{v} K_{v}^{\times p}$. Thus, we have $T=\emptyset$.

Since $\tau$ generates a normal subgroup, we have $\beta_{\emptyset}\left(\Phi^{*}\right)=0$ for $\Phi \neq \varepsilon$ such that $\tau 1_{\Phi}=1_{\Phi}$. Therefore,

$$
\gamma_{\emptyset}\left(\Phi^{*}\right) \geq a_{\Phi}=\alpha\left(\Phi^{*}\right)+\gamma_{\emptyset}\left(\Phi^{*}\right)+\sum_{v_{0} \in P} \delta_{v_{0}}\left(\Phi^{*}\right)-d_{\Phi^{*}}[k: \mathbf{Q}]
$$

Since $C_{\emptyset}^{\tau-1}=\bigoplus_{\tau 1_{\Phi}=1_{\Phi}} 1_{\Phi^{*}} C_{\emptyset}$, we have $\gamma_{\emptyset}\left(\Phi^{*}\right)=0$ for every $\Phi \neq \varepsilon$ such that $\tau 1_{\Phi}=1_{\Phi}$, if $p \nmid h_{K}^{-}$. This proves (1).

Suppose $k=\mathbf{Q}$ and that $M / \mathbf{Q}$ is abelian. By Lemma 4 , the computation of the value of $a_{\varepsilon}$ is reduced to $M_{0}$. Hence, we have $a_{\varepsilon}=0$, because of $p \nmid h_{M_{0}}^{+}$. Let $\Phi$ be an irreducible character such that $\gamma_{\emptyset}\left(\Phi^{*}\right)>0, \Phi \neq \varepsilon$ and $\tau 1_{\Phi}=1_{\Phi}$. Note $\delta_{p}=\omega_{p}$ and $d_{\Phi^{*}}=1$. We observe $a_{\Phi}>0$ from (4.3) if $\gamma_{\emptyset}\left(\Phi^{*}\right) \geq 2$. If $\gamma_{\emptyset}\left(\Phi^{*}\right)=1$, we also observe $a_{\Phi}=0$ if and only if $\alpha\left(\Phi^{*}\right)=\omega_{p}\left(\Phi^{*}\right)=0$. We have $(2)$.

ExAmple 1. Example Let $M$ be the cyclotomic field $\mathbf{Q}\left(\zeta_{m}, \zeta_{p^{n}}\right)$, $p \nmid m, n \geq 2$ and $k$ be the maximal $p$-subfield of $\mathbf{Q}\left(\zeta_{m}\right) / \mathbf{Q}$. We have $K=\mathbf{Q}\left(\zeta_{m}, \zeta_{p}\right)$. If $p$ is not decomposed in $k / \mathbf{Q}$, the class number of $M_{0} k$ is prime to $p$. By Lemma 4 , this means $a_{\varepsilon}=0$. For the following pairs $(m, p)$, 
we have $p \nmid h_{K}^{-}$and that $p$ is not decomposed in $k$ :

\begin{tabular}{|c|c|c|c|c|c|c|}
\hline $\begin{array}{l}(13,3), \\
(15,7), \\
(8,17),\end{array}$ & $\begin{array}{l}(3,13), \\
(16,7), \\
(12,17),\end{array}$ & $\begin{array}{l}(4,13), \\
(28,5), \\
(19,5),\end{array}$ & $\begin{array}{l}(4,17), \\
(20,7), \\
(4,41), \\
(84,5)\end{array}$ & $\begin{array}{l}(3,19), \\
(4,29), \\
(20,11),\end{array}$ & $\begin{array}{l}(5,11), \\
(7,11), \\
(3,43),\end{array}$ & $\begin{array}{l}(35,3) \\
(3,31), \\
(47,3),\end{array}$ \\
\hline
\end{tabular}

These are additional examples to Yamashita [10] for which the Greenberg conjecture are valid for $p$ in the maximal real subfields of $\mathbf{Q}\left(\zeta_{m}, \zeta_{p}\right)$.

\section{REFERENCES}

[1] D.J. Benson, Representations and cohomology I, Cambrige studies in advanced math. 30, Cambrige, 1991.

[2] C.W. Curtis and I. Reiner, Methods of representation theory with applications to finite groups and orders, Vol. I, John Wiley \& Sons, Inc., 1981.

[3] R. Greenberg, On the Iwasawa invariants of totally real number fields, Amer. J. Math., 98 (1976), 263-284.

[4] W. Jehne, On knots in algebraic number theory, J. reine angew. Math., 311/312 (1979), 215-254.

[5] G. Karpilovsky, Group representations, Vol I, Part A and Part B, North-Holland math. studies 175, North-Holland, 1992.

[6] H.W.Leopoldt, Zur Struktur der l-Klassengruppe galoissher Zahlkörper, J. reine angew. Math., 199 (1958), 165-174.

[7] K. Miyake, Central extensions and Schur's multiplicators of Galois groups, Nagoya Math. J., 90 (1983), 137-144.

[8] S. Shirai, On the central class field $\bmod \mathfrak{m}$ of Galois extensions of algebraic number field, Nagoya Math. J., 71 (1978), 61-85.

[9] W. Sinnott, On the Stickelberger ideal and the circular units of a cyclotomic field, Ann. of Math., 108 (1978), 107-134.

[10] H. Yamashita, On the Iwasawa invariants of totally real number fields, manuscripta math., 79 (1993), 1-5.

Faculty of Business Administry and Information Sciences

Kanazawa Gakuin University

Kanazawa, 920-1302 Japan

yamasita@kanazawa-gu.ac.jp 